\title{
Retinal Oxygen Distribution and the Role of Neuroglobin
}

\author{
Paul A. Roberts · Eamonn A. Gaffney · \\ Philip J. Luthert · Alexander J. E. Foss · \\ Helen M. Byrne
}

the date of receipt and acceptance should be inserted later

\begin{abstract}
The retina is the tissue layer at the back of the eye that is responsible for light detection. Whilst equipped with a rich supply of oxygen, it has one of the highest oxygen demands of any tissue in the body and, as such, supply and demand are finely balanced. It has been suggested that the protein neuroglobin $(\mathrm{Ngb})$, which is found in high concentrations within the retina, may help to maintain an adequate supply of oxygen via the processes of transport and storage. We construct mathematical models, formulated as systems of reaction-diffusion equations in one-dimension, to test this hypothesis. Numerical simulations show that Ngb may play an important role in oxygen transport, but not in storage. Our models predict that the retina is most susceptible to hypoxia in the regions of the photoreceptor inner segment and inner plexiform layers, where Ngb has the potential to prevent hypoxia and increase oxygen uptake by $30-40 \%$. Analysis of a simplified model confirms the utility of Ngb in transport and shows that its oxygen affinity $\left(P_{50}\right.$ value $)$ is near optimal for this process. Lastly, asymptotic analysis enables us to identify conditions under which the piecewise linear and quadratic approximations to the retinal oxygen profile, used in the literature, are valid.
\end{abstract}

Paul A. Roberts · Helen M. Byrne

Mathematical Institute, University of Oxford, Andrew Wiles Building, Radcliffe Observatory Quarter, Woodstock Road, Oxford, OX2 6GG, UK, E-mail: p.a.roberts@univ.oxon.org, Tel.: +44-01865-273525, Fax: +44-01865-273583

Department of Computer Science, University of Oxford, Wolfson Building, Parks Road, Oxford, OX1 3QD, UK

Eamonn A. Gaffney

Wolfson Centre for Mathematical Biology, Mathematical Institute, University of Oxford, Andrew Wiles Building, Radcliffe Observatory Quarter, Woodstock Road, Oxford, OX2 6GG, UK

Philip J. Luthert

UCL Institute of Ophthalmology, 11-43 Bath Street, London, EC1V 9EL, UK

Alexander J. E. Foss

Queen's Medical Centre, Department of Ophthalmology, Derby Road, Nottingham, Nottinghamshire, NG7 $2 \mathrm{UH}, \mathrm{UK}$ 
Keywords Asymptotics - Facilitated diffusion - Hypoxia - Oxygen transport · Reaction-diffusion equations

Mathematics Subject Classification (2000) 92B05 · 92C30 · 35K57

\section{Introduction}

The retina is the ocular tissue which detects visual information. Despite being well vascularised, it has a high oxygen demand (Anderson and Saltzman 1964; Anderson 1968; Yu and Cringle 2001; Wangsa-Wirawan and Linsenmeier 2003) placing it in danger of hypoxia (oxygen deprivation). In this paper we construct reaction-diffusion models for retinal oxygen distribution and use them to examine the role of the protein neuroglobin $(\mathrm{Ngb})$ in the prevention of hypoxia.

The retina extends from the optic disc to the ora serrata and has a multilayered structure, consisting of numerous cell-types (see Figure 1(a)). The retina consists of the following layers, beginning with the outermost layer: the retinal pigment epithelium (RPE), the photoreceptor layer, consisting of the outer and inner segments (OSs and ISs), the outer nuclear layer (ONL), the outer plexiform layer (OPL), the inner nuclear layer (INL), the inner plexiform layer (IPL), the ganglion cell layer (GCL), the nerve fibre layer (NFL) and the inner limiting membrane (ILM) (see Figure 1.b)). The outer retina extends from the RPE to the middle of the OPL, where the photoreceptors synapse with bipolar and horizontal cells, whilst the inner retina spans the region between the inner OPL and the ILM. Photoreceptors are responsible for the detection of light and can be characterised as either rods or cones. Following light detection the visual signal is transmitted via the neurons of the inner retina to the optic nerve and then to the brain.

Oxygen is supplied to the retina via two separate vascular systems: the choriocapillaris and the retinal capillaries. The choriocapillaris is a capillary layer which lies directly outward from the RPE and which supplies the outer retina with oxygen. It forms the innermost layer of the choroid and is supplied and drained by blood vessels lying deeper in this tissue. The retinal circulation supplies the inner retina with oxygen and is supplied and drained by the central retinal artery and vein. Throughout most of the mid-peripheral retina, the retinal circulation consists of two capillary layers, one deep and the other superficial (Oyster 1999; Pournaras et al 2008, Kur et al 2012). Therefore, in this paper, we shall consider only the two retinal capillary layer scenario.

Whilst the oxygen distribution across the human retina has not been measured, it has been measured for a variety of mammals including rats (Yu et al 1994, 1999 2000, 2004), rabbits (Stefánsson 1988), cats (Alder et al 1983; Linsenmeier 1986 Linsenmeier and Yancey 1989; Haugh et al 1990; Linsenmeier and Braun 1992 Braun et al 1995, Linsenmeier and Padnick-Silver 2000; Padnick-Silver and Linsenmeier 2003) and monkeys (Yu et al 2005; Birol et al 2007, see also Yu and Cringle 2001; Wangsa-Wirawan and Linsenmeier 2003, Yu and Cringle 2005, for reviews). Under dark conditions (dark adaptation, DA) the photoreceptor ISs consume oxygen at twice the rate at which they consume oxygen under light conditions (light adaptation, LA). As a result, the retinal oxygen profile drops under DA, meaning that 


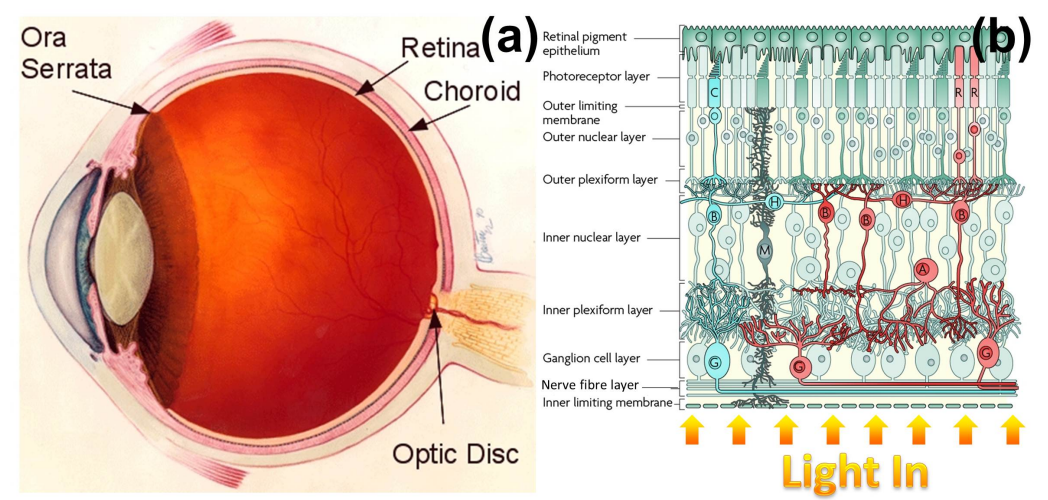

Fig. 1 Diagrams of the human eye and retina. (a) Diagram of the (right) human eye, viewed in the transverse plane. Figure reproduced, with modifications, from http://www.nei.nih.gov/health/coloboma/coloboma.asp, courtesy: National Eye Institute, National Institutes of Health $(\mathrm{NEI} / \mathrm{NIH})$. (b) Diagram of the human retina, showing the retinal layers and cell types. The diagram is oriented such that the top lies outermost and the bottom innermost in the eye. R: rod photoreceptor. C: cone photoreceptor. H: horizontal cell. B: bipolar cell. M: Müller glial cell. A: amacrine cell. G: ganglion cell. Figure reproduced, with permission and modifications, from Swaroop et al (2010)

the retina is more likely to experience hypoxia under DA. Hypoxia is detrimental to cells, both in limiting oxidative metabolism and in increasing the production of reactive oxygen species, upsetting the redox potential and leading to oxidative stress and damage (Kohen and Nyska 2002).

Birol et al (2007) have measured oxygen profiles across the retina of the macaque monkey under DA and LA. The outer retina is normoxic (healthy) under LA and near-hypoxic (just above the hypoxic threshold, see Section 2.1p under DA, whilst the inner retina may be near-hypoxic under either LA or DA, depending upon local retinal capillary density (see Figure 2, where the inner retina is normoxic under LA and near-hypoxic under DA in this case). Since the macaque retina is physiologically similar to that of the human, it is likely that the human retina is also vulnerable to hypoxia, especially under DA. Thus any factor that promotes retinal oxygenation may be vital in preventing or minimising retinal hypoxia and hence cell death.

In 2000, Burmester et al discovered a new vertebrate globin protein which they named neuroglobin because of its preferential expression in the cells of the nervous system, including the retina. Ngb has a similar structure and molecular mass to that of myoglobin $(\mathrm{Mb})$ (Burmester and Hankeln 2009) and, as such, it has been proposed that $\mathrm{Ngb}$, like $\mathrm{Mb}$, may play a role in oxygen transport and storage (Burmester et al 2000; Burmester and Hankeln 2004, 2009).

Despite its oxygen binding properties, it is far from certain that Ngb plays a significant role in oxygen transport and storage, and opinion as to the main function of Ngb remains divided (Burmester and Hankeln 2009).

In this paper we investigate the possible role of $\mathrm{Ngb}$ in oxygen transport and storage in the human retina. The question we wish to address is this: could Ngb 


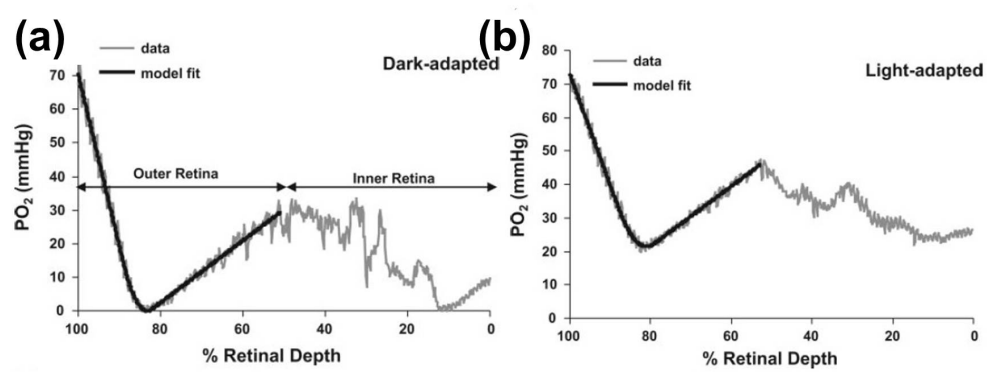

Fig. 2 Retinal oxygen profiles. Graphs show the oxygen distribution across the perifoveal retina of a macaque monkey under DA (a) and LA (b). The retina is normoxic under LA, whilst near-hypoxic regions are present in both the inner and outer retina under DA. Grey lines show the profiles measured using an oxygen-sensitive microelectrode, whilst black lines show a piecewise linear and quadratic approximation fitted to the outer retinal profile. Figure reproduced, with permission, from Birol et al (2007)

prevent tissue hypoxia by transporting oxygen from regions of high oxygen tension to regions of low oxygen tension, or by providing a temporary store of oxygen during a period when oxygen is scarce?

A number of mathematical models for retinal oxygen distribution have been constructed (see for instance, Dollery et al 1969; Linsenmeier 1986; Stefánsson 1988, Haugh et al 1990, Linsenmeier and Braun 1992, Braun et al 1995, Linsenmeier and Padnick-Silver 2000, Cringle and Yu 2002, Yu and Cringle 2002). The majority of these models fit piecewise linear and quadratic polynomials to oxygen profiles measured using oxygen sensitive microelectrodes. They assume that the oxygen concentration is at steady-state and are posed on one-dimensional domains, oriented in a radial direction, across the width of the retina. They assume that the rate of oxygen uptake is constant in each model layer (where model layers incorporate various cellular layers) and so reduce to solving $\frac{\mathrm{d}^{2} c}{\mathrm{~d} x^{2}}=Q$ in each layer, where $c$ is the oxygen concentration, $x$ is the distance across the retina and $Q$ is the rate of oxygen uptake. In those layers in which $Q>0$, the profile is quadratic and in those layers in which $Q=0$, the profile is linear. In Section 4.3 we perform an asymptotic analysis to determine conditions under which the assumption of a constant rate of oxygen uptake is valid, and, in so doing, aim to place existing models on a stronger theoretical foundation.

To the best of our knowledge, only one mathematical model of retinal oxygen distribution incorporating Ngb has been developed (Fago et al 2004b). This model contains three layers spanning the outer retina, between the choriocapillaris and the deep retinal capillaries. Unlike the models mentioned above, a Michaelis-Menten term is used for the rate of oxygen uptake. The proportion of $\mathrm{Ngb}$ molecules in their oxygen bound and unbound states are assumed to be at quasi-steady-state at all times. The model predicts that $\mathrm{Ngb}$ concentrations would have to exceed $100 \mu \mathrm{M}$ to be effective in oxygen storage and to be greater than $300 \mu \mathrm{M}$ to be effective in oxygen transport. Since they assume that the Ngb concentration does not exceed $100 \mu \mathrm{M}$, Fago et al conclude that $\mathrm{Ngb}$ does not play a significant role in either the transport or storage of oxygen. However, based upon our reasoning in the Online Resource, we argue that 
$\mathrm{Ngb}$ concentrations could reach, and, perhaps, significantly exceed, these concentrations in some locations. In what follows we expand Fago et al's model to account for both the inner and outer retina, dropping the quasi-steady-state assumption and exploring the full range of biologically realistic $\mathrm{Ngb}$ concentrations.

The remainder of this paper is structured as follows. In Section 2 we derive timedependent and steady-state models on single and eight layer geometries for the dynamics of oxygen and $\mathrm{Ngb}$ within the retina. In Section 3 we present the results for the single layer model. In Section 4 we present the results for the eight layer model. Lastly, in Section 5, we summarise our findings and discuss possible directions for future research.

\section{Model Formulation}

In this section we derive two models for the oxygen and $\mathrm{Ngb}$ concentrations across the retina. The first model treats the retina as a single cell layer, whilst the second model captures details of its structure and geometry by decomposing it into four cell layers, spread across eight model layers. The single layer model is a toy model, used to ascertain, for a general case, whether $\mathrm{Ngb}$ has the potential to play a significant role in oxygen transport and storage, whilst the second model is used to investigate $\mathrm{Ngb}$ 's role in the human retina.

We develop time-dependent and steady-state versions of each of these models. The time-dependent versions are used to investigate the oxygen storage properties of $\mathrm{Ngb}$, whilst the steady-state versions are used to investigate the oxygen transport properties of $\mathrm{Ngb}$.

$\mathrm{Ngb}$ is a 'hexacoordinate' globin, with the sixth coordination position of the heme group occupied by an internal histidine molecule in the absence of an external ligand (Burmester and Hankeln 2004, 2009, Fago et al 2004b). This means that in order for oxygen to bind to $\mathrm{Ngb}$, the histidine molecule must first unbind from the heme group.

Writing $\mathrm{C}, \mathrm{N}, \mathrm{N}_{\mathrm{H}}$ and $\mathrm{N}_{\mathrm{O}}$ for oxygen, $\mathrm{Ngb}, \mathrm{Ngb}-\mathrm{His}$ and $\mathrm{Ngb}-\mathrm{O}_{2}$ respectively, the reaction scheme may be written as

$$
\begin{array}{r}
\mathrm{N} \underset{\mathrm{k}_{\mathrm{h}}^{-}}{\stackrel{\mathrm{k}_{\mathrm{h}}^{+}}{\rightleftharpoons}} \mathrm{N}_{\mathrm{H}}, \\
\mathrm{N}+\mathrm{C} \underset{\mathrm{k}_{\mathrm{o}}^{-}}{\stackrel{\mathrm{k}_{\mathrm{o}}^{+}}{\rightleftharpoons}} \mathrm{N}_{\mathrm{O}},
\end{array}
$$

where $\mathrm{k}_{\mathrm{h}}^{+}, \mathrm{k}_{\mathrm{h}}^{-}, \mathrm{k}_{\mathrm{o}}^{+}$and $\mathrm{k}_{\mathrm{o}}^{-}$are the forward and reverse rate constants for the different reactions (see Table 1 for values).

\subsection{Single Layer Model}

We begin by formulating a system of four partial differential equations (PDEs) for the concentrations of $\mathrm{O}_{2}, c(x, t)$, Ngb, $n(x, t)$, Ngb-His, $n_{h}(x, t)$ and $\mathrm{Ngb}_{-} \mathrm{O}_{2}, n_{o}(x, t)$, on a one-dimensional domain $x \in(0, L)$ and for time $t \in(0, \infty)$. We choose a domain 


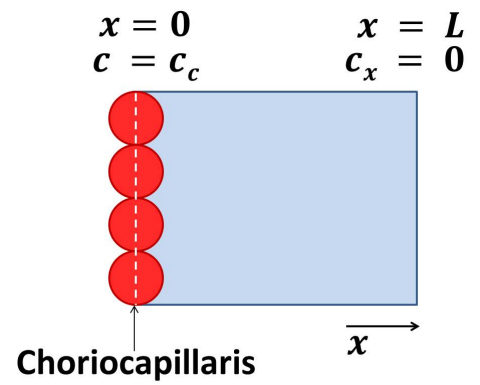

Fig. 3 Single layer model diagram. Oxygen is supplied to the tissue via the choriocapillaris at $x=0$ and the net-flux of oxygen at $x=L$ is zero

orientation perpendicular to the wall of the eye, which cuts across the retinal layers, starting at $x=0$, where the RPE meets the choriocapillaris and ending at $x=L$, at the ILM, where the retina meets the vitreous humour (see Figure 3 .

Using the principle of mass balance and applying the law of mass action to the reaction scheme (1)-2), we derive the following PDE for oxygen,

$$
\frac{\partial c}{\partial t}=\underbrace{D_{o} \frac{\partial^{2} c}{\partial x^{2}}}_{\text {diffusion }}-\underbrace{\frac{Q c}{\gamma+c}}_{\begin{array}{c}
\text { uptake by } \\
\text { retinal tissue }
\end{array}}+\underbrace{k_{o}^{-} n_{o}-k_{o}^{+} n c}_{\begin{array}{c}
\text { unbinding from and } \\
\text { binding to Ngb }
\end{array}},
$$

where $D_{o}$ is the oxygen diffusion coefficient, which is assumed to be constant (Roh et al 1990). The rate of oxygen uptake is known to be near constant above hypoxic levels, but falls rapidly to zero as oxygen levels fall below a critical oxygen concentration (Goldman 2008). Since we are interested in the role of $\mathrm{Ngb}$ in preventing tissue hypoxia, it is important that our oxygen uptake term captures this concentration dependence (Goldman 2008). Thus we use a Michaelis-Menten term for the oxygen uptake (see Wilson et al 1988, Costa et al 1997), where $Q$ is the maximum rate of oxygen uptake by the retinal tissue (achieved in the limit as $c \rightarrow \infty$ ) and $\gamma$ is the Michaelis constant, the concentration of oxygen at which the rate of oxygen uptake is $Q / 2$. The inclusion of oxygen dependence in the uptake term represents an important difference between this model of retinal oxygen distribution and most existing ones (see, for example, Dollery et al 1969; Stefánsson 1988; Haugh et al 1990; Linsenmeier and Braun 1992; Braun et al 1995; Cringle and Yu 2002, Yu and Cringle 2002, an exception being Fago et al 2004b). We also interpret $\gamma$ as being the hypoxic threshold: regions in which oxygen concentrations drop below this value are considered to be hypoxic (Richmond et al 1999; McGuire and Secomb 2001, see also the Online Resource for further details). 
Proceeding similarly, we derive PDEs for $\mathrm{Ngb}(n)$, Ngb-His $\left(n_{h}\right)$, and $\mathrm{Ngb}-\mathrm{O}_{2}$ $\left(n_{o}\right)$,

$$
\begin{aligned}
\frac{\partial n}{\partial t} & =D_{n} \frac{\partial^{2} n}{\partial x^{2}}+k_{h}^{-} n_{h}+k_{o}^{-} n_{o}-k_{h}^{+} n-k_{o}^{+} n c, \\
\frac{\partial n_{h}}{\partial t} & =D_{n} \frac{\partial^{2} n_{h}}{\partial x^{2}}+k_{h}^{+} n-k_{h}^{-} n_{h}, \\
\frac{\partial n_{o}}{\partial t} & =D_{n} \frac{\partial^{2} n_{o}}{\partial x^{2}}+k_{o}^{+} n c-k_{o}^{-} n_{o} .
\end{aligned}
$$

In (4)- (6) we assume that the diffusion coefficients for $\mathrm{Ngb}, \mathrm{Ngb}-\mathrm{His}$ and $\mathrm{Ngb}-\mathrm{O}_{2}$ are identical since they are almost identical in molecular weight and structure (see Keener and Sneyd 1998, where a similar assumption is made about Mb).

We close equations (3)- 6) by imposing the following initial and boundary conditions:

$$
\begin{gathered}
c(x, 0)=c_{\text {init }}(x), n(x, 0)=n_{\text {init }}(x), n_{h}(x, 0)=n_{h_{\text {init }}}(x), n_{o}(x, 0)=n_{\text {oinit }_{\text {it }}}(x), \\
c(0, t)=c_{c}, \frac{\partial n}{\partial x}(0, t)=\frac{\partial n_{h}}{\partial x}(0, t)=\frac{\partial n_{o}}{\partial x}(0, t)=0 \\
\frac{\partial c}{\partial x}(L, t)=\frac{\partial n}{\partial x}(L, t)=\frac{\partial n_{h}}{\partial x}(L, t)=\frac{\partial n_{o}}{\partial x}(L, t)=0 .
\end{gathered}
$$

In $(8) c_{c}$ is the oxygen concentration in that part of the retina which abuts the choriocapillaris. We use a Dirichlet boundary condition at $x=0$, rather than a Robin boundary condition, since the fenestrated capillaries of the choriocapillaris are highly permeable to low molecular weight substances such as oxygen (Törnquist et al 1990). For simplicity, the flux of oxygen at the ILM $(x=L)$ is assumed to be zero. This is reasonable since the rate of oxygen consumption by the vitreous is negligible (Stefánsson 1988, Cringle and Yu 2002). We could alternatively have used Robin boundary conditions; however, in the absence of better data, this added complexity is unjustified. Due to their size, $\mathrm{Ngb}, \mathrm{Ngb}-\mathrm{His}$ and $\mathrm{Ngb}-\mathrm{O}_{2}$ are unable to leave the cells of the retina and, hence, we impose zero-flux boundary conditions for these species at $x=0, L$. See Table 1 for parameter values (where a single value for $Q$ is chosen from the range displayed in the table for any given simulation).

\subsubsection{Non-dimensionalisation}

To simplify the subsequent analysis, we recast the system in non-dimensional form, scaling the independent variables, dependent variables and initial conditions as:

$$
\begin{aligned}
x^{*}=\frac{x}{\tilde{L}}, t^{*}=\frac{D_{o}}{\tilde{L}^{2}} t, \quad c^{*} & =\frac{c}{\tilde{\tilde{c}}}, \quad n^{*}=\frac{n}{\tilde{n}}, \quad n_{h}^{*}=\frac{n_{h}}{\tilde{n}}, \quad n_{o}^{*}=\frac{n_{o}}{\tilde{n}}, \\
c_{\text {init }}^{*}=\frac{c_{\text {init }}}{\tilde{c}}, n_{\text {init }}^{*} & =\frac{n_{\text {init }}}{\tilde{n}}, n_{h_{\text {init }}^{*}}^{*}=\frac{n_{h_{\text {init }}}}{\tilde{n}}, n_{o_{\text {init }}}^{*}=\frac{n_{o_{\text {init }}}}{\tilde{n}},
\end{aligned}
$$

where $\tilde{L}$ is the minimum retinal width, $\tilde{c}$ is a typical oxygen concentration at the choriocapillaris and $\tilde{n}$ is the average Ngb concentration across the retina (see Table 1). (We scale $c$ and $c_{\text {init }}$ with $\tilde{c}$, rather than $c_{c}$, and $x$ and $t$ with $\tilde{L}$, rather than $\mathrm{L}$, so that 


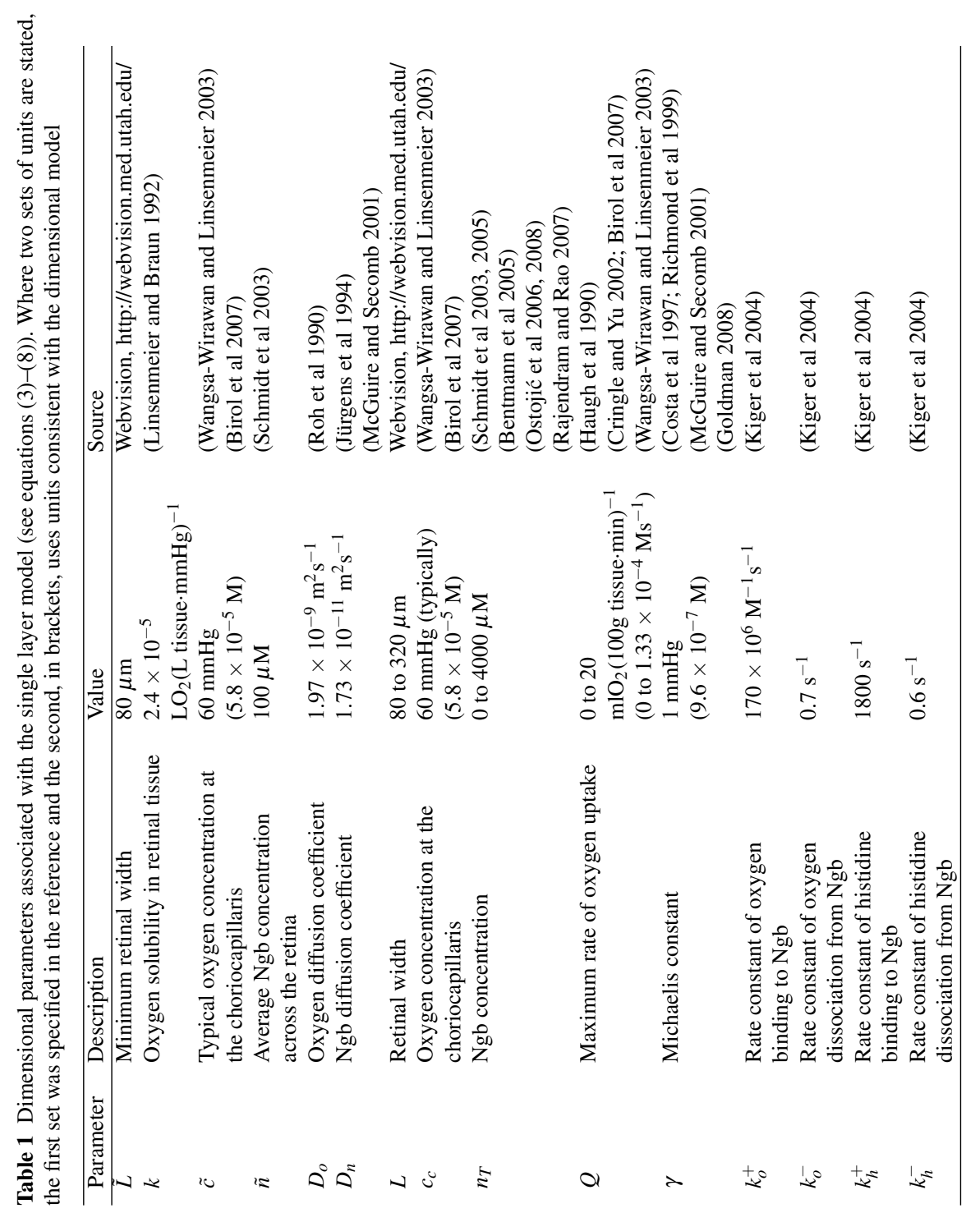


Table 2 Non-dimensional parameters associated with the single layer model (see equations (7)- (12). (Values given to an accuracy of at most three significant figures)

\begin{tabular}{ll}
\hline Parameter & Value \\
\hline$L$ & 1 to 4 \\
$c_{c}$ & 1 \\
$n_{T}$ & 0 to 40 \\
$D$ & $8.78 \times 10^{-3}$ \\
$Q$ & 0 to 7.52 \\
$\gamma$ & 0.017 \\
$\alpha$ & 0.58 \\
$k_{1}$ & 55,200 \\
$k_{2}$ & 3.95 \\
$k_{3}$ & 5,850 \\
$k_{4}$ & 1.95 \\
\hline
\end{tabular}

results using different values of $c_{c}$ and $L$ can be compared more easily.) We define the following non-dimensional parameters:

$$
\begin{aligned}
& L^{*}=\frac{L}{\tilde{L}}, \quad c_{c}^{*}=\frac{c_{c}}{\tilde{c}}, \quad D=\frac{D_{n}}{D_{o}}, \quad Q^{*}=\frac{\tilde{L}^{2}}{D_{o} \tilde{c}} Q, \quad \gamma^{*}=\frac{\gamma}{\tilde{c}}, \\
& \alpha=\frac{\tilde{c}}{\tilde{n}}, k_{1}=\frac{\tilde{L}^{2} \tilde{\tilde{n}}}{D_{o}} k_{o}^{+}, k_{2}=\frac{\tilde{L}^{2} \tilde{\tilde{n}}}{D_{o} \tilde{c}} k_{o}^{-}, k_{3}=\frac{\tilde{L}^{2}}{D_{o}} k_{h}^{+}, k_{4}=\frac{\tilde{L}^{2}}{D_{o}} k_{h}^{-} \text {. }
\end{aligned}
$$

Dropping the stars, the system (3)- 6 becomes

$$
\begin{aligned}
\frac{\partial c}{\partial t} & =\frac{\partial^{2} c}{\partial x^{2}}-\frac{Q c}{\gamma+c}+k_{2} n_{o}-k_{1} n c, \\
\frac{\partial n}{\partial t} & =D \frac{\partial^{2} n}{\partial x^{2}}+k_{4} n_{h}+\alpha k_{2} n_{o}-k_{3} n-\alpha k_{1} n c, \\
\frac{\partial n_{h}}{\partial t} & =D \frac{\partial^{2} n_{h}}{\partial x^{2}}+k_{3} n-k_{4} n_{h}, \\
\frac{\partial n_{o}}{\partial t} & =D \frac{\partial^{2} n_{o}}{\partial x^{2}}+\alpha k_{1} n c-\alpha k_{2} n_{o},
\end{aligned}
$$

with initial and boundary conditions as in (7) and (8). See Table2 for non-dimensional parameter values.

\subsubsection{Steady-State Formulation}

At steady-state, summation and integration of (10)-(12) subject to the zero-flux boundary conditions yields $n+n_{h}+n_{o}=$ constant $=n_{T}$, where $n_{T}$, the total $\mathrm{Ngb}$ concentration, is given. Thus the total quantity of Ngb in its three forms is locally conserved across the domain. We use this identity to eliminate equation (10), writing $n=n_{T}-n_{h}-n_{o}$ in equations (9), (11) and (12), to obtain the steady-state boundary 
value problem:

$$
\begin{aligned}
& 0=\frac{\mathrm{d}^{2} c}{\mathrm{~d} x^{2}}-\frac{Q c}{\gamma+c}+k_{2} n_{o}-k_{1}\left(n_{T}-n_{h}-n_{o}\right) c, \\
& 0=D \frac{\mathrm{d}^{2} n_{h}}{\mathrm{~d} x^{2}}+k_{3}\left(n_{T}-n_{h}-n_{o}\right)-k_{4} n_{h}, \\
& 0=D \frac{\mathrm{d}^{2} n_{o}}{\mathrm{~d} x^{2}}+\alpha k_{1}\left(n_{T}-n_{h}-n_{o}\right) c-\alpha k_{2} n_{o},
\end{aligned}
$$

for $c=c(x), n_{h}=n_{h}(x)$ and $n_{o}=n_{o}(x)$, with boundary conditions as in (8). We note that when $D=0, k_{2} n_{o}-k_{1}\left(n_{T}-n_{h}-n_{o}\right) c=0$ (by equation (15)), so that equation (13) becomes $0=\mathrm{d}^{2} c / \mathrm{d} x^{2}-Q c /(\gamma+c)$. This is the same form taken by the oxygen equation in the absence of Ngb. Therefore, although $D \ll 1$, Ngb diffusion plays an important role and cannot be neglected.

\subsection{Eight Layer Model}

In this section we develop a detailed model to take account of the complex, multilayered structure of the human retina.

We decompose our model retina into eight layers as follows: layer 1 contains the RPE; layer 2, the photoreceptor OSs; layer 3, the photoreceptor ISs; layer 4, the ONL; layer 5, the outer half of the OPL; layer 6, the inner half of the OPL, the INL and the outer half of the IPL; layer 7, the inner half of the IPL, and the outer half of the GCL and NFL and layer 8, the inner half of the GCL and NFL (see Figure 4). (Note that whilst Cringle and Yu (2002) also divide the model retina into 8 layers, their layers are arranged differently, incorporating different tissue layers.) We label the ends of each model layer by $L_{i}$, where $i \in\{1,2, \ldots, 8\}$ and $0<L_{1}<L_{2}<\ldots<L_{8}$.

The number and arrangement of layers have been chosen to allow us to account for the differing oxygen demands of the various retinal layers, to allow the incorporation of vasculature along the boundaries between layers and to allow for the containment of $\mathrm{Ngb}$ within certain layers. The model includes all three vascular layers: the choriocapillaris (on the left-hand boundary of layer 1), the deep retinal capillaries (on the boundary between layers 5 and 6), and the superficial retinal capillaries (on the boundary between layers 7 and 8 , see Figure 4). The deep and superficial retinal capillary layers are both laminar, passing through the OPL and GCL/NFL (Chan et al 2012; Kur et al 2012; Tan et al 2012). 


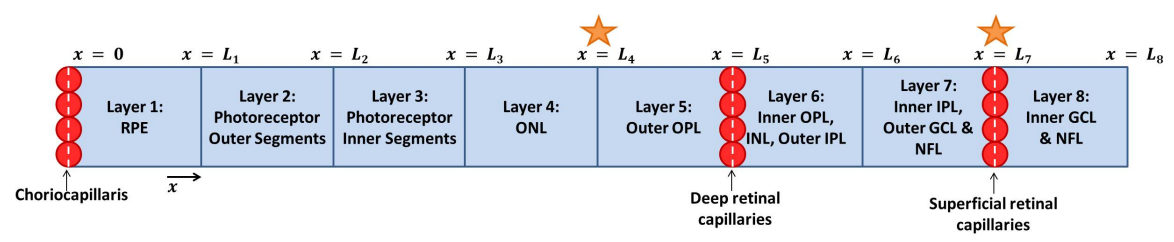

Fig. 4 Eight layer model diagram. Oxygen is supplied to the tissue via the choriocapillaris and retinal capillaries and the net-flux of oxygen at $x=L_{8}$ is zero. The flux of Ngb between layers is zero, except at those boundaries marked with stars, across which the concentration and flux of Ngb is continuous. RPE: retinal pigment epithelium. ONL: outer nuclear layer. OPL: outer plexiform layer. INL: inner nuclear layer. IPL: inner plexiform layer. GCL: ganglion cell layer. NFL: nerve fibre layer

As with the single layer model, the following non-dimensional equations hold in each retinal layer:

$$
\begin{aligned}
\frac{\partial c}{\partial t} & =\frac{\partial^{2} c}{\partial x^{2}}-\frac{Q_{i} c}{\gamma+c}+k_{2} n_{o}-k_{1} n c, \\
\frac{\partial n}{\partial t} & =D \frac{\partial^{2} n}{\partial x^{2}}+k_{4} n_{h}+\alpha k_{2} n_{o}-k_{3} n-\alpha k_{1} n c, \\
\frac{\partial n_{h}}{\partial t} & =D \frac{\partial^{2} n_{h}}{\partial x^{2}}+k_{3} n-k_{4} n_{h}, \\
\frac{\partial n_{o}}{\partial t} & =D \frac{\partial^{2} n_{o}}{\partial x^{2}}+\alpha k_{1} n c-\alpha k_{2} n_{o},
\end{aligned}
$$

where $Q_{i}(1, \ldots, 8)$ denotes the maximum rate of oxygen uptake in each layer. Guided by Roh et al (1990), we suppose that the oxygen diffusion coefficient is constant across the retina and we make the same assumption for the diffusivity of $\mathrm{Ngb}$.

The initial conditions for the eight layer model are the same as in (7). The oxygen boundary conditions for the eight layer model are as follows:

$$
\begin{gathered}
c(0, t)=c_{c}, \frac{\partial c}{\partial x}\left(L_{8}, t\right)=0,\left[c\left(L_{i}, t\right)\right]_{-}^{+}=0, \text { for } i=1, \ldots, 7, \\
{\left[\frac{\partial c}{\partial x}\left(L_{i}, t\right)\right]_{-}^{+}= \begin{cases}0, & \text { for } i=1, \ldots, 4 \text { and } 6, \\
\hat{h}_{i}\left(c\left(L_{i}, t\right)-c_{v}\right), & \text { for } i=5 \text { and } 7 .\end{cases} }
\end{gathered}
$$

where $\hat{h}$ represents the permeability of the retinal capillaries (the dimensional version has the units of permeability, that is $\mathrm{ms}^{-1}$ ) and also accounts for their density, whilst $c_{v}$ is the concentration of oxygen in the retinal capillaries (see the Online Resource for more details). The '-' and '+' subscripts and superscripts indicate that the quantity is evaluated on the left- and right-hand sides of the boundary respectively.

Thus we impose Dirichlet and zero-flux boundary conditions at the left- and righthand ends of the domain respectively, as for the single layer model, with continuity of concentration and flux at all internal boundaries except those at which the capillaries lie, where the flux is discontinuous (see equation (20)).

We impose zero-flux boundary conditions upon $\mathrm{Ngb}, \mathrm{Ngb}-\mathrm{His}$ and $\mathrm{Ngb}-\mathrm{O}_{2}$ at $x=0, L_{1}, L_{5}, L_{6}$ and $L_{8}$ since these represent boundaries between cell layers and $\mathrm{Ngb}$ 
molecules are too large to pass through cell membranes (Ngb only existing intracellularly). As can be seen from Figures 3(a) and 3(b) of the Online Resource, Ngb is highly concentrated within the photoreceptor ISs (layer 3), whilst being absent from the photoreceptor OSs (layer 2) and present in comparatively low concentrations within the ONL (layer 4). It is not known what prevents Ngb from diffusing from layer 3, into layers 2 and 4; however, it may be that the narrow connecting cilium prevents $\mathrm{Ngb}$ from diffusing between layers 2 and 3. Therefore, we also impose zero-flux boundary conditions upon $\mathrm{Ngb}, \mathrm{Ngb}-\mathrm{His}$ and $\mathrm{Ngb}-\mathrm{O}_{2}$ at $x=L_{2}$ and $L_{3}$, in order to maintain the localisation of Ngb within the ISs. The concentration and flux of $\mathrm{Ngb}, \mathrm{Ngb}-\mathrm{His}$ and $\mathrm{Ngb}-\mathrm{O}_{2}$ are continuous across $x=L_{4}$ and $L_{7}$ since these model boundaries occur within cell layers, there being nothing to physically prevent these species from diffusing across these boundaries.

\subsubsection{Steady-State Formulation}

As with the single layer model, the following non-dimensional equations define the steady-state distributions for $c=c(x), n_{h}=n_{h}(x)$ and $n_{o}=n_{o}(x)$ in each retinal layer:

$$
\begin{aligned}
& 0=\frac{\mathrm{d}^{2} c}{\mathrm{~d} x^{2}}-\frac{Q_{i} c}{\gamma+c}+k_{2} n_{o}-k_{1}\left(n_{T_{i}}-n_{h}-n_{o}\right) c, \\
& 0=D \frac{\mathrm{d}^{2} n_{h}}{\mathrm{~d} x^{2}}+k_{3}\left(n_{T_{i}}-n_{h}-n_{o}\right)-k_{4} n_{h}, \\
& 0=D \frac{\mathrm{d}^{2} n_{o}}{\mathrm{~d} x^{2}}+\alpha k_{1}\left(n_{T_{i}}-n_{h}-n_{o}\right) c-\alpha k_{2} n_{o},
\end{aligned}
$$

where $Q_{i}$ and $n_{T_{i}}(1, \ldots, 8)$ denote the maximum rate of oxygen uptake in each layer and the total $\mathrm{Ngb}$ concentration in each layer respectively. The boundary conditions are the same as for the time-dependent eight layer model (see (20) above).

The dimensional and non-dimensional parameters associated with the eight layer model are presented in Tables 3 and 4 respectively (where the upper and lower values for $Q_{3}$ correspond to DA and LA respectively). Those parameters used in the eight layer model which are the same as in the single layer model are stated in Tables 1 and 2.

\section{Single Layer Model Results}

\subsection{Numerical Solutions to the Steady-State Problem - Oxygen Transport}

We begin by presenting results for the steady-state single layer problem, where we investigate the role of $\mathrm{Ngb}$ in oxygen transport. The equations for the single layer model, (8) and (13)-(15), were solved using the finite element method (FEM). We validated our FEM solutions to the steady-state problem by checking that they matched the steady-state solutions to the time-dependent problem, (7)-(12), solved using the method of lines. In what follows, all variable and parameter values are given in their non-dimensional form, unless stated otherwise. 


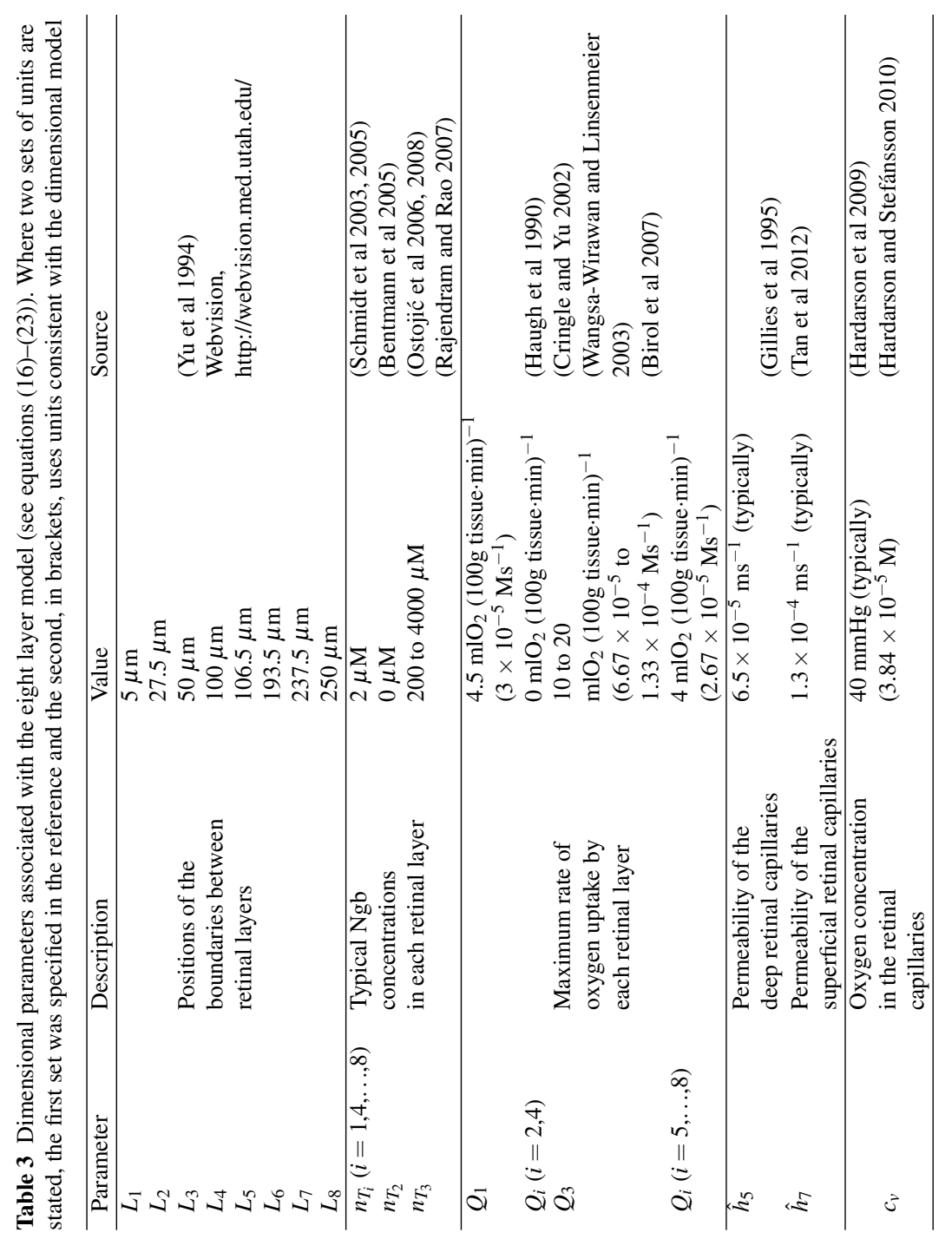


Table 4 Non-dimensional parameters associated with the eight layer model (see equations [16- 23]). (Values given to an accuracy of at most three significant figures)

\begin{tabular}{ll}
\hline Parameter & Value \\
\hline$L_{1}$ & 0.063 \\
$L_{2}$ & 0.34 \\
$L_{3}$ & 0.63 \\
$L_{4}$ & 1.25 \\
$L_{5}$ & 1.33 \\
$L_{6}$ & 2.42 \\
$L_{7}$ & 2.97 \\
$L_{8}$ & 3.13 \\
\hline$n_{T_{i}}(i=1,4, \ldots, 8)$ & 0.02 \\
$n_{T_{2}}$ & 0 \\
$n_{T_{3}}$ & 2 to 40 \\
\hline$Q_{1}$ & 1.69 \\
$Q_{i}(i=2,4)$ & 0 \\
$Q_{3}$ & 3.76 to 7.52 \\
$Q_{i}(i=5, \ldots, 8)$ & 1.50 \\
\hline$\hat{h}_{5}$ & 2.64 \\
$\hat{h}_{7}$ & 5.28 \\
\hline$c_{v}$ & 0.67 \\
\hline
\end{tabular}

Figure 5 shows how increasing the total concentration of $\mathrm{Ngb}$ in the retina, $n_{T}$, affects the steady-state oxygen distribution. Increased $\mathrm{Ngb}$ levels result in a minor decrease in the oxygen concentration in the well oxygenated region of the tissue near $x=0$, but a significant increase in the oxygen concentration near $x=L$. The decrease in oxygen concentration near the choriocapillaris is insignificant since oxygen is rich here; however, the increase in oxygen concentration near $x=L$ represents a significant alleviation of hypoxia. Indeed, the oxygen concentration does not pass beneath the hypoxic threshold, $\gamma$, for $n_{T} \geq 12$, where $n_{T}=16$ is the maximum biologically realistic value for a domain of this length.

\subsection{Analysis of a Simplified Single Layer Model}

In this section we develop and analyse a simplified version of the single layer model at steady-state. We use the simplified model to investigate Ngb's effect upon the domain length that can be supported under normoxic conditions and upon the minimum oxygen concentration when oxygen levels do not become hypoxic. (Keener and Sneyd 1998. Chapter 2, perform a similar analysis for a problem involving the facilitated diffusion of oxygen by $\mathrm{Mb}$ in a slab reactor, the main difference being that their equations lack an oxygen uptake term.)

To simplify our analysis we make two changes: we replace the Michaelis-Menten term, $Q c /(\gamma+c)$, with a step function $Q H(c-\gamma)$, so that the rate of oxygen uptake is equal to zero when $c<\gamma$ and $Q$ when $c \geq \gamma$. Secondly, we set $\tilde{n}=58 \mu \mathrm{M}$, so that $\alpha=1$. This alternative choice for the non-dimensionalisation does not affect the behaviour of the system, it merely simplifies the analysis presented below (allowing cancellation of the kinetic terms). The non-dimensional parameters $n_{T}, k_{1}$ and $k_{2}$ 


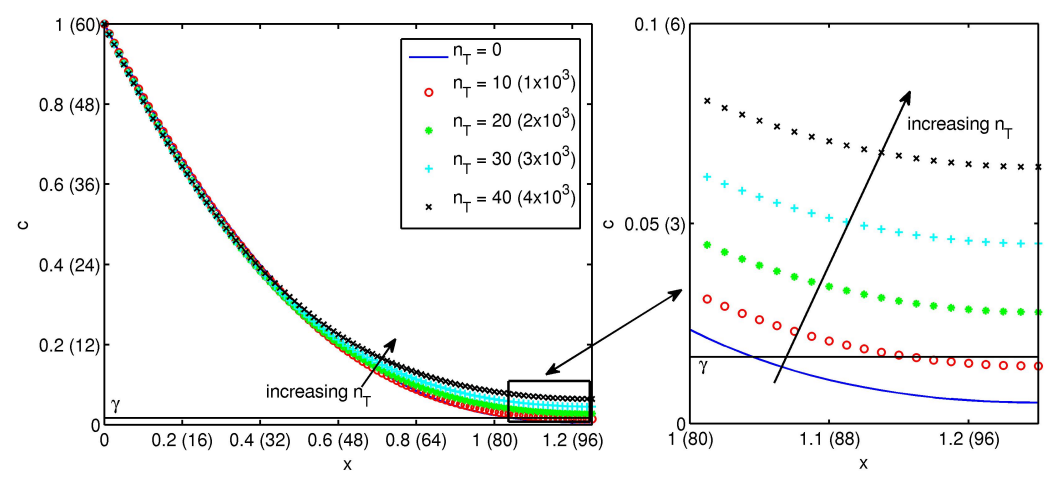

Fig. 5 Simulation results from the steady-state single layer model. As the total concentration of Ngb, $n_{T}$, is increased, the steady-state oxygen concentration at $x=L$ increases above the hypoxic threshold, $\gamma$. Equations (8) and [13 - 15 were solved using the FEM with 101 mesh points. Dimensional values are given in brackets for $x(\mu \mathrm{m}), c(\mathrm{mmHg})$ and $n_{T}(\mu \mathrm{M})$. Parameter values: $L=1.25(100 \mu \mathrm{m}), c_{c}=1(60$ $\mathrm{mmHg})$ and $Q=1.88\left(3.33 \times 10^{-5} \mathrm{Ms}^{-1}\right)$. Remaining parameter values as in Table 2

Table 5 Non-dimensional parameters associated with the simplified single layer model (see equations (24)-26). Only those parameter values that change have been listed, all other parameter values remain as in Table 2 (Values given to an accuracy of at most three significant figures).

\begin{tabular}{ll}
\hline Parameter & Value \\
\hline$n_{T}$ & 0 to 69.4 \\
$\alpha$ & 1 \\
$k_{1}$ & 31,800 \\
$k_{2}$ & 2.27 \\
\hline
\end{tabular}

also change, attaining the values stated in Table 5 , whereas all other parameter values remain as in Table 2

Following these simplifications, equations $[13-15]$ take the form

$$
\begin{aligned}
& 0=\frac{\mathrm{d}^{2} c}{\mathrm{~d} x^{2}}-Q H(c-\gamma)+k_{2} n_{o}-k_{1} n c, \\
& 0=D \frac{\mathrm{d}^{2} n_{h}}{\mathrm{~d} x^{2}}+k_{3} n-k_{4} n_{h}, \\
& 0=D \frac{\mathrm{d}^{2} n_{o}}{\mathrm{~d} x^{2}}+k_{1} n c-k_{2} n_{o},
\end{aligned}
$$

where $n=n_{T}-n_{o}-n_{h}$. We impose the boundary conditions $(8)$ to close the system.

We begin by considering the case where the retina becomes hypoxic, such that $c(x) \leq \gamma$ for $x_{c} \leq x \leq L$. Adding equations (24) and [26, applying the zero-flux boundary condition for $n_{o}$ at $x=0$ and integrating between 0 and $x$ supplies

$$
0=\frac{\mathrm{d} c}{\mathrm{~d} x}(x)-\frac{\mathrm{d} c}{\mathrm{~d} x}(0)+D \frac{\mathrm{d} n_{o}}{\mathrm{~d} x}(x)-Q \underbrace{\int_{0}^{x} H(c-\gamma) \mathrm{d} \tilde{x}}_{=\min \left(x, x_{c}\right)},
$$


where $x_{c}$ is the maximum domain length that can be supported under normoxic conditions, such that $c\left(x_{c}\right)=\gamma$. Applying the zero-flux boundary conditions for $c$ and $n_{o}$ at $x=L$, we find that $\frac{\mathrm{d} c}{\mathrm{~d} x}(0)=-Q x_{c}$. Rearranging equation 27], we obtain the following expression for $J(x)$, the flux of oxygen (in its bound and unbound forms):

$$
J(x)=-\left(\frac{\mathrm{d} c}{\mathrm{~d} x}+D \frac{\mathrm{d} n_{o}}{\mathrm{~d} x}\right)=Q\left(x_{c}-\min \left(x, x_{c}\right)\right) .
$$

Integrating equation (28) between 0 and $x_{c}$, we obtain the following expression:

$$
J_{A}=\int_{0}^{x_{c}} J(x) \mathrm{d} x=\left(c_{c}-\gamma\right)+D\left(n_{o}(0)-n_{o}\left(x_{c}\right)\right)=\frac{Q x_{c}^{2}}{2},
$$

where $J_{A}$ is a conserved quantity equal to the area under the curve $J(x)$. We seek an expression for $x_{c}$, in order to determine the contribution of $\mathrm{Ngb}$ to the domain length that can be supported under normoxia. We proceed by deriving leading order approximations for $n_{o}(0)$ and $n_{o}\left(x_{c}\right)$ in terms of $c(0)=c_{c}$ and $c\left(x_{c}\right)=\gamma$ respectively. Expanding the dependent variables in regular power series in terms of the small parameter $\varepsilon=0.1$ and substituting into equations 24$]-26$, we retain the (dominant) leading order terms and neglect higher order terms.

We begin by re-scaling the variables and parameters, fixing $\varepsilon=0.1$ to separate the various scales. We scale $c=\varepsilon c^{*}$, since $c$ is of this order across most of the retina (see, for instance, Figure 5), $D=\varepsilon^{2} D^{*}, \gamma=\varepsilon^{2} \gamma^{*}, k_{1}=\varepsilon^{-4} k_{1}^{*}$ and $k_{3}=\varepsilon^{-3} k_{3}^{*}$.

In Appendix A we show that $n$ is bounded above by $\alpha k_{2} n_{T} / k_{3}$. Using the alternative non-dimensionalisation adopted in this section, this becomes $k_{2} n_{T} / k_{3} \approx \varepsilon^{3} n_{T}$ (where the changes in $\alpha$ and $k_{2}$ cancel). Whilst $n_{o}$ and $n_{h}$ vary across the domain, they are of the same order of magnitude, and so, at leading order, they satisfy the conservation relation $n_{o}+n_{h}=n_{T}$. Assuming that $2 \leq n_{T} \leq 69.4$, the Ngb terms can be scaled in one of two ways, depending upon the size of $n_{T}$ :

- Scaling 1: for $2 \leq n_{T}<7$ we scale $n=\varepsilon^{3} n^{*}$, where $n_{h}=O(1), n_{o}=O(1)$ and $n_{T}=O(1)$.

- Scaling 2: for $7 \leq n_{T} \leq 69.4$ we scale $n=\varepsilon^{2} n^{*}, n_{h}=\varepsilon^{-1} n_{h}^{*}, n_{o}=\varepsilon^{-1} n_{o}^{*}$ and $n_{T}=\varepsilon^{-1} n_{T}^{*}$.

In both cases $n / n_{T}=O\left(\varepsilon^{3}\right), n_{h} / n_{T}=O(1)$ and $n_{o} / n_{T}=O(1)$. In what follows we work with the first scaling, noting that the leading order solution under the second scaling is the same. Applying Scaling 1 to equations 24-26 we obtain:

$$
\begin{aligned}
& 0=\varepsilon \frac{\mathrm{d}^{2} c^{*}}{\mathrm{~d} x^{2}}-Q H^{*}\left(c^{*}-\gamma^{*}\right)+k_{2} n_{o}-k_{1}^{*} n^{*} c^{*}, \\
& 0=\varepsilon^{2} D^{*} \frac{\mathrm{d}^{2} n_{h}}{\mathrm{~d} x^{2}}+k_{3}^{*} n^{*}-k_{4} n_{h}, \\
& 0=\varepsilon^{2} D^{*} \frac{\mathrm{d}^{2} n_{o}}{\mathrm{~d} x^{2}}+k_{1}^{*} n^{*} c^{*}-k_{2} n_{o},
\end{aligned}
$$

where $H^{*}\left(c^{*}-\gamma^{*}\right)=H(c-\gamma)$. Upon dropping the stars, equations (31) and 32 provide

$$
k_{3} n-k_{4} n_{h}=0, \quad k_{1} n c-k_{2} n_{o}=0,
$$




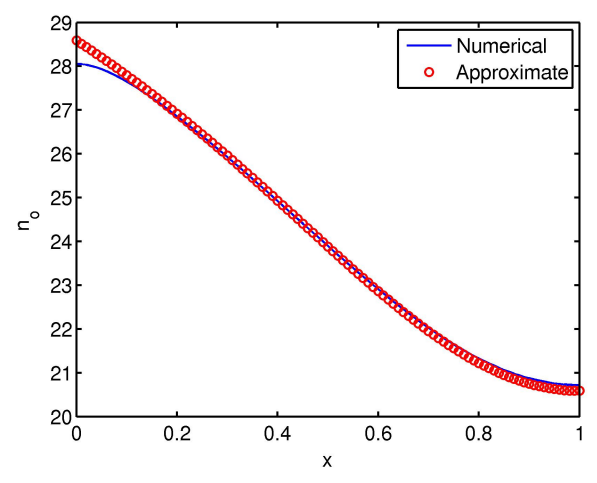

Fig. 6 Graph showing that the approximate solution for $n_{o}$ matches closely with the numerical solution. The numerical solution was obtained by solving equations 8 and 24 - 26 using the FEM, with 101 mesh points. The approximate solution was obtained using the numerical solution for $c$ in equation 34 . Parameter values: $L=1, c_{c}=1, n_{T}=34.7$ and $Q=1.50$. Remaining parameter values as in Tables 2 and 5 Parameters and variables are in their unscaled form

at leading order. Substituting the second of these equations into (30), the dominant balance involves the diffusion and uptake terms. For $x \geq x_{c}, Q H(c-\gamma)=0$, so that, after applying $c\left(x_{c}\right)=\gamma$, together with the zero-flux boundary condition at $x=L$, we find that $c(x)=\gamma$ for $x \in\left[x_{c}, L\right]$. For $x \in\left[0, x_{c}\right), c>\gamma$, so that $Q H(c-\gamma)>0$, and we must scale $x=\varepsilon^{\frac{1}{2}} x^{*}$ to attain a dominant balance between the diffusion and uptake terms, resulting in a quadratic oxygen profile in this region. Applying this scaling to equations (31) and (32) retrospectively, we see that the leading order equations in (33) are preserved. Formally, there is also a boundary layer at $x=0$, to allow us to satisfy the boundary conditions there (though there is no sharp change in the solution in this region).

We use (33), together with the leading order conservation relation $n_{h}+n_{o}=n_{T}$, to derive an expression for $n_{o}$ in terms of $c$ as follows

$$
n_{o}=\frac{n_{T} c}{P_{50}+c}, \text { where } P_{50}:=\frac{k_{2} k_{3}}{k_{1} k_{4}} .
$$

$P_{50}$ is the oxygen concentration at which exactly half of the Ngb molecules are in their oxygen bound form, that is at which $n_{o}=n_{T} / 2$ ( $=n_{h}$ at leading order). This expression for $P_{50}$ differs from that used by Kiger et al (2004), where $P_{50}=k_{2}\left(k_{3}+\right.$ $\left.k_{4}\right) /\left(k_{1} k_{4}\right)$. Kiger et al's expression can be obtained by considering the unscaled, spatially homogeneous versions of equations $24-26$ with $Q=0$. However, since $k_{4} \ll k_{3}$ (in their unscaled form), the difference between these two expressions is small. As Figure 6 reveals, the approximate solution for $n_{o}$ in equation (34) matches closely with the numerical solution.

Substituting for $n_{o}$ from (34) into 29) we obtain

$$
J_{A}=\left(c_{c}-\gamma\right)(1+\underbrace{\frac{P_{50} D n_{T}}{\left(P_{50}+c_{c}\right)\left(P_{50}+\gamma\right)}}_{\text {Ngb contribution }})=\frac{Q x_{c}^{2}}{2} \text {. }
$$



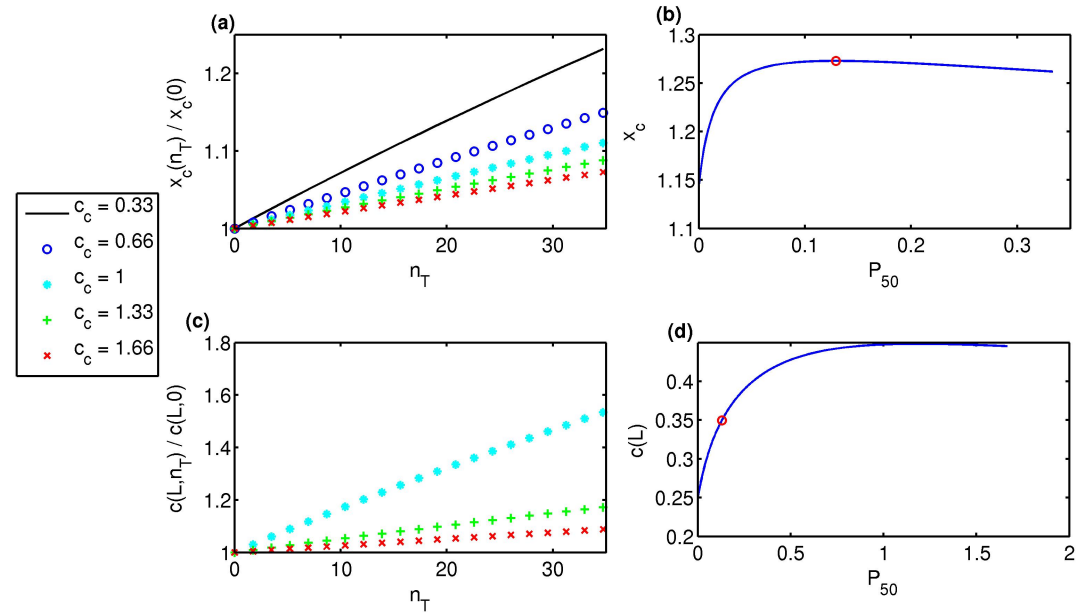

Fig. 7 Graphs showing results for the simplified model. Graphs (a) and (c) show the proportional increase in $x_{c}$ and $c(L)$ respectively, due to the action of $\mathrm{Ngb}$, the effect being more pronounced for lower values of $c_{c}$. Graphs (b) and (d) show the effect of $P_{50}$ upon $x_{c}$ and $c(L)$ respectively. The value of the dependent variable at $P_{50}=\sqrt{\gamma c_{c}}$ is marked with a circle in each case. The value of $x_{c}$ is maximal at $P_{50}=\sqrt{\gamma c_{c}}$; however, $c(L)$ takes its maximum value for some $P_{50}>\sqrt{\gamma c_{c}}$. Graphs (a) and (b) were obtained using solutions to (36) and graphs (c) and (d) were obtained using solutions to (39). Parameter values: $Q=1.50$, (c)-(d) $L=1$, (b) and (d) $c_{c}=1$ and $n_{T}=34.7$. Remaining parameter values as in Tables 2 and 5 The key on the left applies to graphs (a) and (c) only

Rearranging equation (35), we may derive an expression for $x_{c}$, the maximum domain length that can be supported under normoxic conditions, as follows

$$
x_{c}=\sqrt{\frac{2\left(c_{c}-\gamma\right)}{Q}\left(1+\frac{P_{50} D n_{T}}{\left(P_{50}+c_{c}\right)\left(P_{50}+\gamma\right)}\right)} .
$$

If $n_{T}=\gamma=0$ then (36) reduces to the expression derived by Dollery et al (1969) for the maximum tissue length that can be supported by a single vascular bed. It can be seen both from equation (36) and from Figure 7 7 a) that $\mathrm{Ngb}$ increases the size of $x_{c}$. For $c_{c}=1$ and $n_{T}=34.7$ (our predicted maximum $\mathrm{Ngb}$ concentration for domains of this length, assuming a maximal retinal width of $320 \mu \mathrm{m}$, and that $\mathrm{Ngb}$ is absent from all other retinal layers), $x_{c}$ is $11 \%$ longer than it would be in the absence of $\mathrm{Ngb}$. Further, Ngb makes a proportionally larger contribution to $x_{c}$ as $c_{c}$ decreases (see Figure 7(a)), for instance, when $n_{T}=34.7$ and $c_{c}=0.33, x_{c}$ is $23 \%$ larger than it would be in the absence of $\mathrm{Ngb}$.

Differentiating equation (36) with respect to $P_{50}$, we find that $x_{c}$ achieves a global maximum at $P_{50, \max }=\sqrt{\gamma c_{c}}$. Figure 7 (b) shows the dependence of $x_{c}$ upon $P_{50}$, with the maximum value of $x_{c}$, at which $P_{50}=P_{50, \max }$, marked with a circle. It can be seen that the curve is fairly flat in the vicinity of $P_{50, \max }$ such that $x_{c}$ remains near maximal for a small decrease, or even quite a large increase, in $P_{50}$ away from $P_{50, \text { max }}$. Further, $x_{c}$ decreases sharply as $P_{50}$ approaches zero for $P_{50}<0.05$. This result is surprising 
since it is usually assumed that Ngb would be a more effective oxygen transporter if it had a lower $P_{50}$ value: our analysis reveals that as $P_{50}$ decreases below $P_{50, \text { max }}, \mathrm{Ngb}$ will become less effective in oxygen transport. For $c_{c}=1, P_{50, \max }=0.129=7.44$ $\mu \mathrm{M}=7.75 \mathrm{mmHg}$, which is within the range of measured values in the literature (see Table 3 of the Online Resource).

So far we have assumed that the retina becomes hypoxic at some location, $x=$ $x_{c}<L$. If instead, oxygen levels are normoxic throughout the domain, that is $x_{c}>L$, then we may derive an expression for $c(x=L)$, the minimum oxygen concentration. Equation (28) becomes

$$
J(x)=-\left(\frac{\mathrm{d} c}{\mathrm{~d} x}+D \frac{\mathrm{d} n_{o}}{\mathrm{~d} x}\right)=Q(L-x) .
$$

Integrating (37) between 0 and $L$ and substituting for $n_{o}$ from (34) we obtain

$$
J_{A}=\left(c_{c}-c(L)\right)\left(1+\frac{P_{50} D n_{T}}{\left(P_{50}+c_{c}\right)\left(P_{50}+c(L)\right)}\right)=\frac{Q L^{2}}{2} .
$$

This equation can be rearranged to give the following expression for $c(L)$ :

$$
c(L)=\frac{-A+\sqrt{A^{2}-4 B}}{2},
$$

where we have chosen the positive root to satisfy the condition that $c(L)=c_{c}$ when $Q=n_{T}=0$ (see equation 38 ), and

$$
A=P_{50}+\frac{Q L^{2}}{2}+\frac{P_{50} D n_{T}}{P_{50}+c_{c}}-c_{c}, \quad B=P_{50}\left(\frac{Q L^{2}}{2}-\frac{D n_{T} c_{c}}{P_{50}+c_{c}}-c_{c}\right) .
$$

Figure 7(c) shows that $\mathrm{Ngb}$ acts to increase $c(L)$, making a proportionally larger contribution for lower $c_{c}$. Indeed, for $L=1, c_{c}=1, n_{T}=34.7$ (our predicted maximum $\mathrm{Ngb}$ concentration for domains of length $L=1$ ) and $Q=1.50, c(L)$ is $53 \%$ higher than what it would be in the absence of $\mathrm{Ngb}$.

Plotting $c(L)$ against $P_{50}$ shows that the maximum value of $c(L)$ does not correspond with $P_{50}=\sqrt{\gamma c_{c}}$, but instead lies at a $P_{50}$ significantly above this value (this relationship was found to hold for all combinations of parameter values tested, see Figure $7(\mathrm{~d})$ ). It may be that the optimum $P_{50}$ lies somewhere above $\sqrt{\gamma c_{c}}$, since $x_{c}$ decreases slowly as $P_{50}$ increases beyond $\sqrt{\gamma c_{c}}$, but $c(L)$ increases more rapidly. In both cases a decrease in $P_{50}$ below $\sqrt{\gamma c_{c}}$ is disadvantageous, counter to the prevailing assumption that lower $P_{50}$ values improve Ngb's effectiveness in oxygen transport.

\subsubsection{Comparing Numerical and Approximate Solutions}

In deriving an analytical solution for $x_{c}$ we have made two simplifying assumptions: (i) that the Michaelis-Menten term for oxygen uptake may be approximated by a step function and (ii) that $n_{o}$ can be approximated as a function of $c$ (equation (34)). In this section we compare the approximate analytical solutions with the numerical solutions to the full (equations $(8)$ and $(13)-(15)$ ) and simplified (equations 
(8) and (24)-(26) problems. When solving the simplified problem numerically, we replace the step function in 24), $H(c-\gamma)$, with the modified hyperbolic function, $\frac{1}{2}(\tanh ((c-\gamma) / \delta)+1)$, where $\delta=0.001$, so as to avoid the numerical difficulties resulting from a discontinuity in the governing equations.

Figure 8 (a) compares the approximate solution to the numerical solution to the full problem, for the proportional increase in $x_{c}$ due to $\mathrm{Ngb}$ (the numerical solution to the simplified problem provides a closer match to the approximate solution, but is omitted for clarity), whilst Figure 8 (b) compares the results for the approximate solution and the numerical solutions to the simplified and full models, for the dependence of $x_{c}$ upon $P_{50}$. The difference between results is at most $O(\varepsilon)$ (where $\varepsilon=0.1$ ) in all cases, as expected for a leading order approximation. The numerical solution to the simplified problem achieves its maximum for $P_{50}$ slightly above $\sqrt{\gamma c_{c}}$, whilst the numerical solution to the full problem achieves its maximum for $P_{50} \approx 0.28=16.7$ $\mathrm{mmHg}$. This is approximately twice $\sqrt{\gamma c_{c}}=0.13=7.7 \mathrm{mmHg}$, and lies at the upper end of the range of measured values in the literature (see Table 3 of the Online Resource, where the bracketed $P_{50}$ values are the ones consistent with the parameters used in our model). Unlike the approximate and simplified numerical solutions, the prediction of $x_{c}$ from the full numerical solution initially decreases as $P_{50}$ increases from 0 (this result was verified using the Matlab routine pdepe to solve the timedependent problem (7)-(12), allowing it to settle to steady-state). Whilst this results in a local maximum at $P_{50}=0$, the global maximum remains at $P_{50, \text { max }, \text { numeric }}>0$. Therefore, we have a reasonable agreement between the numerical solutions to the full and simplified problems and our approximations.

\section{Eight Layer Model Results}

\subsection{Numerical Solutions to the Steady-State Problem - Oxygen Transport}

As with the single layer model, we solved the steady-state eight layer model, 20, [23), using the FEM. We validated our FEM solutions to the steady-state problem by checking that they matched the steady-state solution to the time-dependent problem, (7) and (16)-(20), solved using the method of lines. We now return to the nondimensionalisation described in Section 2 (as opposed to that adopted in Section 3.2).

Figure 9 shows the oxygen profile across a healthy human retina under LA (dotted curve) and DA (solid curve) in the absence of Ngb. The boundaries between model layers are shown by vertical lines and the layers are labelled 1-8, whilst a horizontal line marks the hypoxic threshold, $\gamma$. The only difference between LA and DA in our model is that the rate of oxygen uptake by the photoreceptor ISs, $Q_{3}$, under DA is double that under LA. As a result, the oxygen concentration in the outer retina (layers 1-5) and in layer 6, is significantly lower under DA than under LA, whereas the oxygen concentration in layers 7 and 8 remains relatively unaffected.

The parameters used to generate this plot are either taken directly from the literature, or estimated from the literature, except for the permeabilities of the deep and superficial retinal capillaries, $\hat{h}_{5}$ and $\hat{h}_{7}$. These are chosen to yield oxygen profiles similar to those measured in the rat (see Yu et al 1994. Figure 2(a)) and macaque (see 

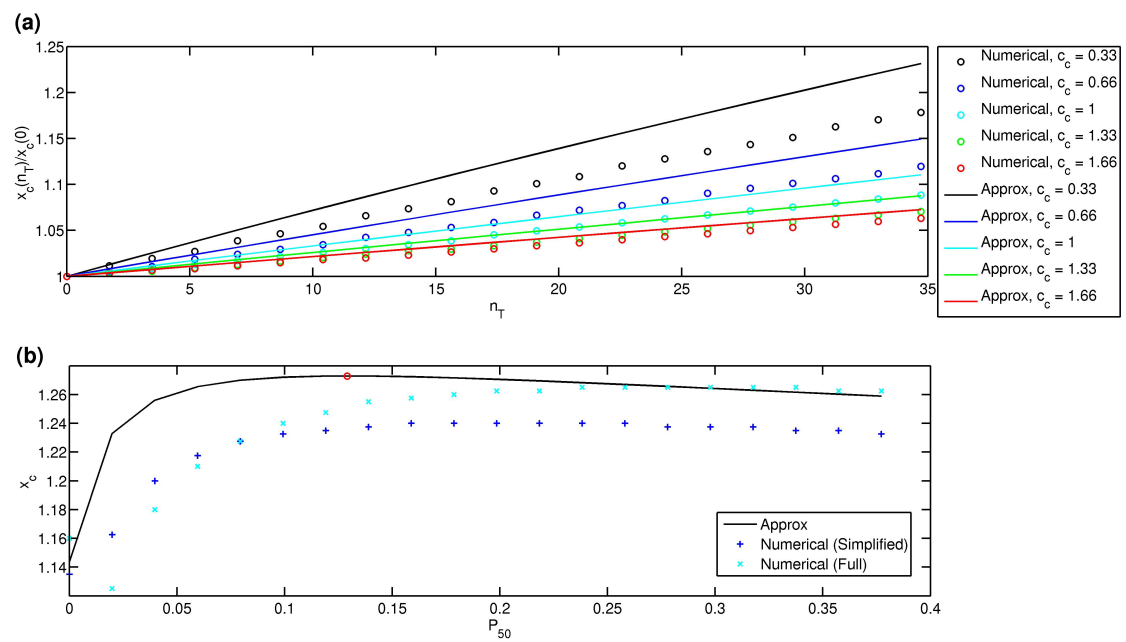

Fig. 8 Graphs comparing numerical and approximate solutions to the single layer model. Graph (a) compares the results for the proportional increase in $x_{c}$, due to the action of $\mathrm{Ngb}$, from the approximation (36) and the solution to the full problem $[8$ and $[13-15$. In general the approximate solution overestimates the numerical solution, particularly for small values of $c_{c}$. Graph (b) compares the results for the effect of $P_{50}$ upon $x_{c}$ from the approximation [36, the solution to the simplified problem [8] and 24, -26 and the solution to the full problem 8 and $(13)-\sqrt{15}$; the value of $x_{c}$ at $P_{50}=\sqrt{\gamma c_{c}}$ is marked with a circle. The numerical solutions to the simplified and full problems lie mostly beneath the approximate solution. The numerical solutions to the full and simplified problems were obtained using the FEM with 801 mesh points. Parameter values: $L=2$ and $Q=1.50$; (b) $c_{c}=1$ and $n_{T}=34$.7. Remaining parameter values as in Tables 2 and 5

Figure 2) retinas (which are physiologically and biochemically similar to the human retina), whilst satisfying the constraint $\hat{h}_{7}=2 \hat{h}_{5}$, since the superficial retinal capillary bed has twice the density of the deep retinal capillary bed (Tan et al 2012, see the Online Resource for more details). Therefore, our simulations of the retinal oxygen profile in humans constitute testable predictions.

Since the oxygen profile in the inner retina of the macaque may descend to near hypoxic levels (see Figure 2(a)), we choose the permeabilities such that the inner retinal oxygen profile descends beneath the hypoxic threshold in the absence of $\mathrm{Ngb}$ under DA. This allows us to test whether Ngb could prevent hypoxia in such a scenario, raising the oxygen profile to levels similar to those seen in the healthy macaque. Further, as we vary $\hat{h}_{5}$ and $\hat{h}_{7}$ between $0.2-3.1$ and $0.4-6.2$ respectively (under DA, results not shown) we find that the outer retina will remain normoxic provided $c_{c}$ is not significantly reduced, whilst the inner retina (layers 6-8) will remain hypoxic, achieving its minimum in layer 6 , as is the case in Figure 9

We note that whilst Figure 9 matches well with the oxygen profile seen in rats (see, for example, Yu and Cringle (2002)), our model places the minimum inner retinal oxygen concentration at a position outward from that measured in the macaque (see Figure 2). However, in the absence of measurements for the retinal oxygen pro- 


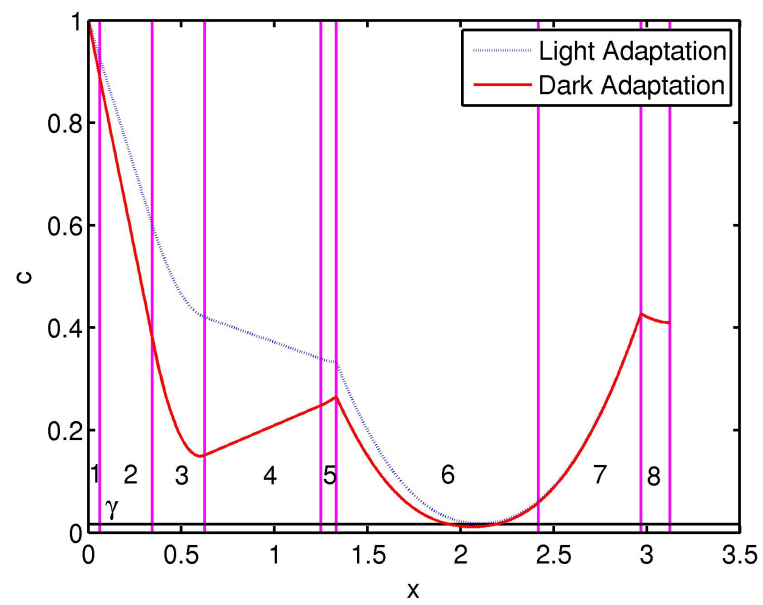

Fig. 9 Simulation results from the eight layer model showing the oxygen distribution in the healthy human retina under LA and DA in the absence of $\mathrm{Ngb}$. The spatial extent of the different layers is depicted by the vertical lines, whilst the hypoxic threshold, $c=\gamma$, is denoted by a horizontal line. The oxygen concentration in the outer retina (layers 1-5) and layer 6 is significantly lower under DA due to the increased rate of oxygen uptake by the photoreceptor ISs. Equations 20, 23 were solved using the FEM with 501 mesh points. Under LA $Q_{3}=3.76$, whereas under DA $Q_{3}=7.52$. In both cases $n_{T_{i}}=0(i=1, \ldots, 8)$. Remaining parameter values as in Tables 2 and 4

file in humans with which to compare our results, it is not clear that any modification of the model would be an improvement.

In Figure 10 we show that Ngb may prevent inner retinal hypoxia. The parameters chosen are the same as those for Figure 9 under DA (for which the inner retina is hypoxic in the absence of $\mathrm{Ngb}$ ). The solid curve shows the oxygen profile in the absence of $\mathrm{Ngb}$ (this is the same as the solid curve in Figure 9) and the dotted curve shows the oxygen profile in the presence of $\mathrm{Ngb}$, when $\mathrm{Ngb}$ is most heavily concentrated in layers 3, 6, 7 and 8. This Ngb distribution corresponds with that found in the retina of the rat by Bentmann et al (2005) (see Online Resource). The average Ngb concentration across the retina in this simulation is approximately $432 \mu \mathrm{M}$, which is larger than the average of $100-200 \mu \mathrm{M}$ measured in the mouse retina by Schmidt et al (2003). However, since $\mathrm{Ngb}$ is confined to the cytosol (the fluid compartment of cells excluding the nucleus and organelles) of retinal cells, it may be that $\mathrm{Ngb}$ could reach an average concentration in the cytosol much greater than $200 \mu \mathrm{M}$, without the average concentration of $\mathrm{Ngb}$ across the whole retina exceeding this value. As such, the Ngb concentration in our model should be interpreted as the concentration in the cytosol. It can be seen that the addition of $\mathrm{Ngb}$ raises the oxygen profile in the inner retina, increasing the minimum in layer 6 so that it no longer passes beneath the hypoxic threshold, $c=\gamma$. (In this case, our model predicts an average inner retinal oxygen level of $8.5 \mathrm{mmHg}$, which is within the range of values measured by Birol et al (2007) in the rhesus monkey.) This corresponds to an increase in the minimum 


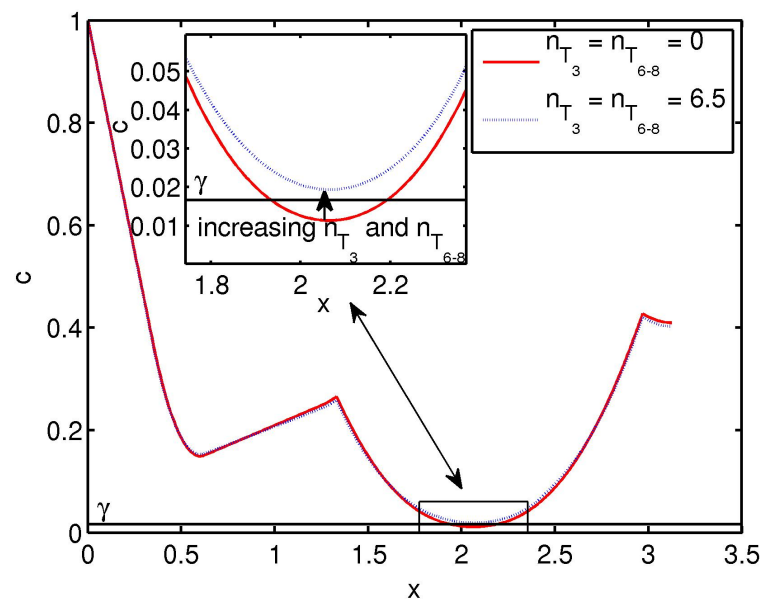

Fig. 10 Simulation results from the eight layer model showing how the presence of Ngb in the inner retinal layers may prevent inner retinal hypoxia under DA. Key: $\mathrm{Ngb}$ absent (solid curve: $n_{T_{i}}=0, i=1, \ldots, 8$ ); Ngb present (dotted curve: $n_{T_{1}}=n_{T_{4}}=n_{T_{5}}=0.02, n_{T_{2}}=0$ and $n_{T_{3}}=n_{T_{6}}=n_{T_{7}}=n_{T_{8}}=6.50$ ). Equations 20] -23 were solved using the FEM with 501 mesh points. Parameter values: $Q_{3}=7.52$. Remaining parameter values as in Tables 2 and 4

rate of oxygen uptake in the inner retina from $40.1 \%$ to $53.3 \%$ of $Q_{6}$, the maximum rate of oxygen uptake in this region (see equation (21)).

In Figure 11 we decrease $c_{c}, c_{v}, \hat{h}_{5}$ and $\hat{h}_{7}$ (the oxygen concentration at the choriocapillaris and in the retinal capillaries, and the permeabilities of the deep and superficial retinal capillaries respectively) so that the outer retina is hypoxic in the absence of $\mathrm{Ngb}$, more closely representing the macaque retina under DA (Birol et al 2007). It is found that if $\mathrm{Ngb}$ is concentrated primarily in the photoreceptor ISs $\left(L_{2}<x<L_{3}\right)$, then it may prevent outer retinal hypoxia (raising the outer retinal minimum oxygen concentration to $1.1 \mathrm{mmHg}$, which falls within the range $1.3 \pm 0.2 \mathrm{mmHg}$ measured by Yu et al (2005) in the macaque). This corresponds to an increase in the minimum rate of oxygen uptake in the outer retina from $36.8 \%$ to $51.8 \%$ of $Q_{3}$, the maximum rate of oxygen uptake in this region (see equation (21). We remark that the inner retina is hypoxic in both cases since there is insufficient $\mathrm{Ngb}$ in this region. In this case, the average concentration of $\mathrm{Ngb}$ across the retina is approximately $362 \mu \mathrm{M}$.

There is some variability between measured retinal oxygen distributions, particularly within the inner retina. Therefore, whilst it is beyond the scope of the present work, we note that a detailed parameter sensitivity analysis would provide a valuable extension, allowing us to determine the range of scenarios under which $\mathrm{Ngb}$ is effective in preventing or alleviating hypoxia. 


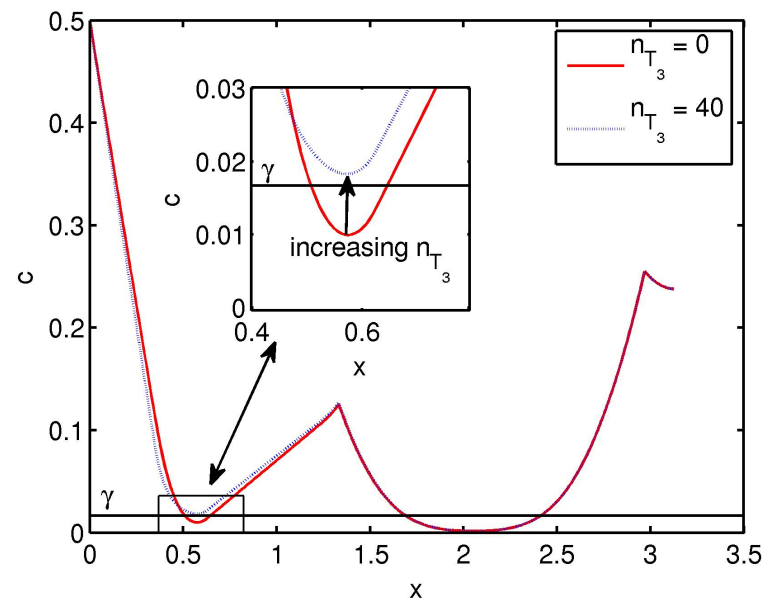

Fig. 11 Simulation results from the eight layer model showing how the presence of Ngb in the photoreceptor ISs may prevent outer retinal hypoxia under DA. Key: Ngb absent (solid curve: $n_{T_{i}}=0, i=1, \ldots$, 8); $\mathrm{Ngb}$ present (dotted curve: $n_{T_{1}}=n_{T_{i}}=0.02, n_{T_{2}}=0, n_{T_{3}}=40, i=4, \ldots, 8$ ). Equations 20]-23] were solved using the FEM with 501 mesh points. Parameter values: $c_{c}=0.5, Q_{3}=7.52, \hat{h}_{5}=2.03, \hat{h}_{7}=4.06$ and $c_{v}=0.5$. Remaining parameter values as in Tables 2 and 4

\subsection{Numerical Solutions to the Time-Dependent Problem - Oxygen Storage}

Simulations were run for both the single and eight layer models to determine the maximum period of time for which $\mathrm{Ngb}$ could buffer the retina against hypoxia, following either an increase in the rate of oxygen uptake, $Q$, or a decrease in the oxygen supply, $c_{c}$ or $c_{v}$ (results not shown). It was found that $\mathrm{Ngb}$ could prevent hypoxia for no more than a few seconds and hence we conclude that it is unlikely to play a significant role in oxygen storage.

\subsection{Asymptotic Analysis of the Neuroglobin-free Problem}

In this section we perform an asymptotic analysis of the Ngb-free, steady-state problem, in order to validate the piecewise linear and quadratic approximations to the oxygen profile used by Braun et al (1995); Cringle and Yu(2002) and others. For this purpose, we consider the parameter values to be of the same order of magnitude as those used in Figure 9, as these represent standard conditions for the human retina. In the absence of $\mathrm{Ngb}$ there is no reason to distinguish between layers 6 and 7, since the purpose of the boundary between these layers was to ensure that Ngb could not pass between them. Therefore, we now group layers 6 and 7 together, labelling this new merged layer, 'layer $6^{*}$, and relabel 'layer 8 ' as 'layer $7^{*}$ '. The details of the analysis can be found in Appendix B.

To summarise, we have found that $c(x)$ is quadratic in layers $1,3,5$ and $7^{*}$ at leading order, that it is linear in layers 2 and 4 , and may be approximated by quadratics 
in layer $6^{*}$. That is,

$$
c(x)= \begin{cases}\frac{Q_{i} x^{2}}{2}+A_{i} x+B_{i}, & \text { for } i \in\left\{1,3,5,7^{*}\right\}, \\ A_{i} x+B_{i}, & \text { for } i \in\{2,4\}, \\ \frac{Q_{6^{*}}}{2}\left(L_{5}-x\right)^{2}+\sqrt{2 Q_{6^{*}} c_{L}}\left(L_{5}-x\right)+c_{L}, & \text { left-hand, layer } 6^{*}, \\ \left(1+\frac{Q_{6^{*}}\left(x_{\min }-x\right)^{2}}{2\left(\gamma+c_{\min }\right)}\right) c_{\min }, & \text { central, layer } 6^{*}, \\ \frac{Q_{6^{*}}}{2}\left(x-L_{6^{*}}\right)^{2}+\sqrt{2 Q_{6^{*}} c_{R}}\left(x-L_{6^{*}}\right)+c_{R}, & \text { right-hand, layer } 6^{*},\end{cases}
$$

where $x_{\min }$ and $c_{\min }$, the position and size respectively, of the local minimum in layer $6^{*}$, are given by equations 66 and 67 respectively in Appendix $\mathrm{B}, c_{L}=c\left(L_{5}\right)$, $c_{R}=c\left(L_{6^{*}}\right)$ and the $A_{i} \mathrm{~s}$ and $B_{i} \mathrm{~s}$ are constants of integration. The equations contain 14 unknowns $\left(A_{1} \ldots A_{5}, A_{7^{*}}, B_{1} \ldots B_{5}, B_{7^{*}}, c_{L}\right.$ and $\left.c_{R}\right)$, which can be found by imposing the 14 boundary conditions upon the external and internal boundaries $\left(c(0)=c_{c}\right.$, $c_{x}\left(L_{7^{*}}\right)=0$ and the concentration and flux conditions across the internal boundaries at $x=L_{i}$, for $\left.i=1, \ldots, 6^{*}\right)$. The approximation is continuous, except within layer $6^{*}$, where it is not possible to obtain continuity between the left-hand, central and right-hand approximations. The accuracy of the approximation can be improved using iteration, resulting in slight modifications to the values of some of the unknowns listed above, as described in Appendix B.

Figure 12 compares the approximate solution with the finite element solution to the full problem using the same parameter values as in Figure 9 for the dark adapted case. The approximate solution agrees reasonably well with the FEM solution, though it underestimates it, particularly in layers 3 and 4 . The approximate value of $x_{\min }$, $x_{\min }^{a p p}=2.0625$ (4 d.p.), is very close to the FEM value, $x_{\min }^{F E M}=2.0625$ (4 d.p.), with an error of $O\left(10^{-5}\right)$, whilst the approximate value of $c_{\min }, c_{\min }^{a p p}=0.0091$ (4 d.p.), is less close to the FEM value, $c_{\text {min }}^{F E M}=0.0114$ (4 d.p.), though it is of the same order of magnitude, with an error of $O\left(10^{-3}\right)$.

This analysis shows that the piecewise linear and quadratic profiles used by Braun et al (1995); Cringle and Yu (2002) and others are valid, provided the oxygen concentration is $O(1)$ or $O(\varepsilon)$. If the oxygen concentration drops to $O\left(\varepsilon^{2}\right)$, that is, if it becomes hypoxic or near-hypoxic, a quadratic approximation is valid; however, the coefficients of the quadratic are not the same as those based on the assumption of constant oxygen uptake used in the literature. In this case the coefficients must be modified, as outlined above. We also note that when fitting curves to measured oxygen profiles, the value of $Q$ in those layers where $c=O\left(\varepsilon^{2}\right)$ will be underestimated when using a constant oxygen uptake assumption since $c /(\gamma+c)$ is significantly less than unity in these regions.

\section{Discussion}

The retina is one of the most oxygen hungry tissues in the human body, such that, despite being well vascularised, it is frequently at risk of hypoxia. In this paper we have investigated the part that may be played by the protein Ngb in the prevention of hypoxia, through its roles in oxygen transport and storage. 


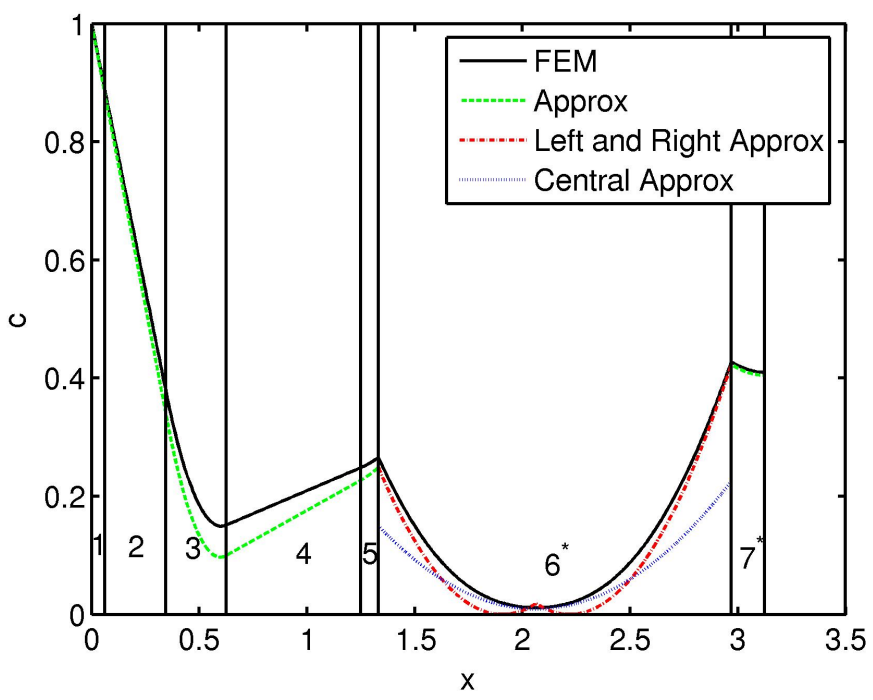

Fig. 12 Comparison of the approximate solution with the FEM solution to the full problem. The approximate solution matches well with the FEM solution, though it is less accurate in layers 3 and 4 . The left-hand solution in layer $6^{*}$ is plotted from $x=L_{5}$ to $x=x_{\min }$, the right-hand solution is plotted from $x=x_{\min }$ to $x=L_{6^{*}}$ and the central solution is plotted throughout layer $6^{*}$. The spatial extent of the different layers is depicted by the vertical lines. Parameter values are the same as those used in Figure 9 for the dark adapted case

Single and eight layer models of the retina were derived in one spatial dimension for both the steady-state and time-dependent problems associated with oxygen transport and storage respectively (Section 2).

The single layer model is a toy model, such that, whilst it does not capture the complex physiology and cellular compartmentalisation of the retina, it provides a useful starting point for investigating the role of $\mathrm{Ngb}$ in oxygen transport and storage for a more generic scenario. Examination of the steady-state single layer model (Section 3.1) demonstrated that $\mathrm{Ngb}$ acts by removing oxygen from oxygen rich regions and redistributing it to oxygen poor regions, with the potential to eliminate or alleviate hypoxia.

Steady-state solutions of the eight layer model revealed that Ngb may play an important role in oxygen transport in the human retina (Section 4.1). Whilst the effect of $\mathrm{Ngb}$ on the oxygen profile is subtle, an increase in the oxygen concentration through the hypoxic threshold, $\gamma$, may result in a significant increase in the rate of oxygen uptake (as much as 30-40\%) since the Michaelis-Menten curve is steepest in a neighbourhood of $c=\gamma$. It was found that $\mathrm{Ngb}$ may prevent inner retinal hypoxia, provided it is sufficiently concentrated in this region. Similarly, if the outer retina is vulnerable to hypoxia, then outer retinal hypoxia may be avoided, provided $\mathrm{Ngb}$ is present in sufficient quantities in the photoreceptor ISs.

Whilst our model oxygen profiles may differ from the, as yet unmeasured, human retinal oxygen profiles and whilst there is some inter-individual variability, the eight 
layer model shows that Ngb could in principle prevent or alleviate hypoxia in the human retina, provided Ngb is appropriately distributed. Detailed measurements of the cytosolic Ngb concentration in different cellular layers of the retina are needed in order to determine whether $\mathrm{Ngb}$ is present in the quantities that we suggest are necessary for $\mathrm{Ngb}$ to play a significant role in oxygen transport.

We expect $\mathrm{Ngb}$ to be most highly concentrated in those layers of the retina that are most vulnerable to hypoxia. This would mean that $\mathrm{Ngb}$ distribution would differ between species, as retinal oxygen distribution differs between species, and perhaps within individual eyes, as oxygen supply and demand vary with eccentricity. The most closely related species in which retinal oxygen and $\mathrm{Ngb}$ distributions have been measured are the mouse and rat, where it was found that Ngb is indeed most highly concentrated in those regions where oxygen is most scarce (Yu et al 1994, Schmidt et al 2003, 2005). Further studies are needed to confirm whether this holds true in other species. The only study to date to examine the relationship between Ngb distribution and eccentricity in the human retina found no differences in the patterns of immunoreactivity for $\mathrm{Ngb}$ between the central and peripheral retina (Ostojić et al 2008); however, this does not exclude the possibility that, whilst Ngb may be present within the same retinal layers across the retina, it may be distributed differently between those layers for different eccentricities. Further experimental work is required to test this prediction.

We also note that $\mathrm{Ngb}$ in one region of the retina can play a protective role in preventing hypoxia in another region, even if $\mathrm{Ngb}$ cannot pass between these two regions. This is evident in Figure 10, where Ngb in layer $7(x \in[2.42,2.97])$ raises the oxygen concentration on the left-hand side of the layer, so that it is at a higher concentration as it enters layer 6, a region vulnerable to hypoxia. Therefore, whilst Bentmann et al (2005) observe that the distribution of cytoglobin (a protein that has also been proposed to play a role in oxygen transport within the retina, Burmester et al 2002, Trent and Hargrove 2002) is unrelated to mitochondria distribution and oxygen consumption, this does not imply that cytoglobin cannot play a role in oxygen transport and storage, since it does not need to be colocalised with mitochondria containing regions in order to increase the oxygen supply to these regions. Having said this, the compartmentalisation of $\mathrm{Ngb}$ within the retina does reduce its capacity to transport oxygen across the retina, since oxygen-bound $\mathrm{Ngb}$ will often meet a barrier before reaching an oxygen poor region where it can deposit its oxygen. In other words, if $\mathrm{Ngb}$ is confined within a narrow region across which the drop in oxygen concentration is small, then Ngb will have a less pronounced effect than if allowed to move across a larger region where the drop in oxygen concentration is large and hence across which the drop in Ngb oxygen saturation is large.

$\mathrm{Ngb}$ must be present in high concentrations and the duration of unfavourable parameter fluctuations brief in order for $\mathrm{Ngb}$ to be of significant benefit in oxygen storage (Section 4.2). If there was a scenario in which $c_{c}$ or $Q$ oscillated on the time scale of a few seconds, and particularly if the periods of recovery were longer than the periods of challenge, then Ngb could prevent or reduce hypoxia to a significant degree (results not shown); however, as far as we are aware, no such scenario exists. Thus, whilst the model parameters may differ between individuals, it is highly unlikely that any situation could obtain in which $\mathrm{Ngb}$ could store oxygen on the timescale 
of minutes to hours that would be necessary for it to play a significant role in the prevention of hypoxia via oxygen storage.

Therefore we conclude that Ngb may play an important role in oxygen transport, but it is unlikely to play an important role in oxygen storage.

The conclusion that Ngb may play an important role in oxygen transport is further supported by our analysis of the simplified single layer model (Section 3.2). This demonstrated that $\mathrm{Ngb}$ increases the maximum domain length that can be supported under normoxia, $x_{c}$, and the minimum oxygen concentration in a normoxic domain, $c(L)$. It was found that the proportional contribution of $\mathrm{Ngb}$ to these quantities is larger for lower values of $c_{c}$, the oxygen concentration at the choriocapillaris. This is consistent with the idea that $\mathrm{Ngb}$ has an important role in oxygen transport, particularly when the oxygen supply is limited.

The analysis also indicated that $x_{c}$ is maximised for an oxygen affinity of $P_{50, \max }=$ $\sqrt{\gamma c_{c}}$, decreasing sharply as $P_{50} \rightarrow 0$ and decreasing gradually initially as $P_{50}$ increases past its optimum value. This trend was confirmed by the numerical solution to the full problem; however, the $P_{50}$ value for which $x_{c}$ is maximised was shown to be approximately twice that predicted by the analysis. It is surprising that the optimum $P_{50}$ value should be so high, since it is assumed in the literature that a lower $P_{50}$ value will result in more efficient oxygen transport. For $c_{c}$ in the range $20-100 \mathrm{mmHg}$ (the maximum expected range in humans and other mammals), the numerical solution to the full problem predicts that $P_{50, \text { max }, \text { numeric }}=9.5-17.9( \pm 2) \mathrm{mmHg}$, whereas a $P_{50}$ corresponding to that of $\mathrm{Mb}(0.9-2.2 \mathrm{mmHg})$ is generally thought to be preferable in the literature (see for instance Burmester and Hankeln 2004, 2009). Four of the eight measured values of $\mathrm{Ngb} P_{50}$ presented in Table 3 of the Online Resource (relevant $P_{50}$ values in brackets) fall within our predicted range, meaning that the oxygen affinity has the value one would expect if $\mathrm{Ngb}$ plays a role in oxygen transport. Therefore, the fact that $\mathrm{Ngb}$ has a higher $P_{50}$ value than $\mathrm{Mb}$ is advantageous, rather than problematic, for oxygen transport (suggesting that natural selection may have tuned this parameter to its present value). Indeed, given the shallow gradient of the curve in Figure 8 b) for $P_{50}>P_{50, \text { max, numeric }}$, oxygen transport would remain near-optimal even for $P_{50}$ values significantly higher than $P_{50, \text { max ,numeric }}$. We note also that these results suggest that the $P_{50}$ value of $\mathrm{Ngb}$ may differ between species, varying according to the square root of the average oxygen concentration at the capillaries. Further experimental work is required to test this prediction.

A tractable analytical expression for the $P_{50}$ value which maximises $c(L)$ in a normoxic domain could not be obtained; however, numerical solution with a range of parameter values suggests that the optimal $P_{50}$ value lies consistently above that which optimises $x_{c}$, that is above $P_{50, \text { max,numeric }}$. This suggests that the overall optimal $P_{50}$ value may lie a little above $P_{50, \text { max }, \text { numeric }}$.

In addition to illustrating the general principle that Ngb's oxygen transport properties may be optimised for a higher $\mathrm{P}_{50}$ value than that taken by $\mathrm{Mb}$, the scenarios captured by the simplified single layer model may occur at several points in the human retina. For instance, in both layers 3 and 6, the oxygen profile may drop from a normoxic concentration at one end of the layer, to hypoxic levels within the layer, whilst in layer 8 , the oxygen concentration remains normoxic across the layer, satisfying a zero-flux boundary condition at $x=L_{8}$. 
Lastly, asymptotic analysis of the steady-state Ngb-free problem (Section 4.3 , demonstrated that the assumption of a constant rate of oxygen uptake is valid, provided the oxygen concentration is $O(\varepsilon)$ (where $\varepsilon=0.1$, corresponding to $\approx 6 \mathrm{mmHg}$ ), or larger. This places existing models for retinal oxygen distribution on a stronger theoretical foundation. If the oxygen concentration drops to hypoxic or near-hypoxic levels, at $O\left(\varepsilon^{2}\right)$, then the oxygen profile can still be approximated using a quadratic, but the coefficients will be different from those which result from the constant rate of oxygen uptake assumption.

This work could be extended in several ways. The magnitude of the histidine dissociation rate, $k_{h}^{-}$, could be made to vary according to the oxygen concentration, so that Ngb's oxygen affinity decreases in hypoxia and increases in normoxia, to examine the extent to which this enhances the oxygen transport and storage properties of $\mathrm{Ngb}$ (see the Online Resource and Hamdane et al 2003, for details). Models could be developed to examine the potential role of $\mathrm{Ngb}$ at other eccentricities within the human retina, or in other species, by adjusting the parameters relating to geometry, oxygen supply and oxygen demand. Also, cytoglobin could be included in the models, to determine whether it significantly augments the oxygen transport and storage properties of $\mathrm{Ngb}$. The model could also be extended to 2D or 3D, and more detailed descriptions of cell metabolism and blood flow incorporated.

In conclusion, although Ngb's transport function results in only a minor shift in the oxygen profile, this can result in a significant rise in oxygen uptake and may be enough to prevent hypoxia if supply and demand are finely balanced. $\mathrm{Ngb}$ is unlikely to be important for oxygen storage. Contrary to the prevailing assumption, the fact that $\mathrm{Ngb}$ has a higher $P_{50}$ value than $\mathrm{Mb}$ is not evidence against its role in oxygen transport, and indeed many of the $P_{50}$ values measured thus far are near optimal for oxygen transport. Finally, the assumption of a piecewise constant rate of oxygen uptake, used in previous studies, is valid, provided oxygen levels do not drop to $O\left(\varepsilon^{2}\right)$.

Acknowledgements We gratefully acknowledge the Engineering and Physical Sciences Research Council (EPSRC) in the UK for funding through a studentship at the Systems Biology programme of the University of Oxford's Doctoral Training Centre P.A.R.

Appendix A: Placing a Bound on the Concentration of Pentacoordinate Neuroglobin

It was found in all single and eight layer model simulations that the concentration of pentacoordinate $\mathrm{Ngb}, n$, at any given point in space is significantly lower than that of hexacoordinate $\mathrm{Ngb}, n_{h}$, or oxygen bound $\mathrm{Ngb}, n_{o}$, at that point. In this appendix we derive a bound on $n$ to explain why this is the case.

Beginning with the time-dependent equation for $n$, equation 10 , we can re-write it in the form

$$
\frac{\partial n}{\partial t}=D \frac{\partial^{2} n}{\partial x^{2}}+f(x, t)-\left(\frac{1}{\kappa}+g(x, t)\right) n,
$$

where $1 / \kappa=k_{3}, f(x, t)=k_{4} n_{h}+\alpha k_{2} n_{o} \geq 0$ and $g(x, t)=\alpha k_{1} c \geq 0$. Whether we are using the single or the eight layer model, this equation will be defined on a finite 
domain, which we can denote as $x \in[0, L]$ without loss of generality. As usual, we impose zero-flux boundary conditions at either end of the domain.

Consider the function $M=M(t)$ which satisfies

$$
\frac{\mathrm{d} M}{\mathrm{~d} t}=\left(\max _{x, t}(f(x, t))+\beta\right)-\frac{1}{\kappa} M,
$$

where the constant $\beta>0$ is arbitrarily small. This can be written as

$$
\frac{\mathrm{d} M}{\mathrm{~d} t}=F-\frac{1}{\kappa} M,
$$

where $F=\left(\max _{x, t}(f(x, t))+\beta\right)>f(x, t) \geq 0$ is a constant. We close the system by imposing the initial condition

$$
M(0)=\max _{x}(n(x, 0)) .
$$

Solving this initial-value problem using the integrating factor method we obtain

$$
M(t)=e^{-\frac{t}{\kappa}} M(0)+\kappa F\left(1-e^{-\frac{t}{\kappa}}\right) .
$$

Taking the limit as $t$ tends to infinity we find that

$$
\lim _{t \rightarrow \infty} M(t)=\kappa F .
$$

Let $u(x, t):=n(x, t)-M(t)$, then

$$
\frac{\partial u}{\partial t}-D \frac{\partial^{2} u}{\partial x^{2}}=(f-F)-\frac{u}{\kappa}-g n,
$$

from equations (42) and (44). Since $(f-F)<0$ and $g n \geq 0$ we have that

$$
\frac{\partial u}{\partial t}-D \frac{\partial^{2} u}{\partial x^{2}}+\frac{u}{\kappa}<0,
$$

and therefore

$$
\frac{\partial}{\partial t}\left(e^{\frac{t}{\kappa}} u\right)-D \frac{\partial^{2}}{\partial x^{2}}\left(e^{\frac{t}{\kappa}} u\right)<0 .
$$

Defining $v(x, t):=e^{\frac{t}{\kappa}} u(x, t)$, we have that

$$
\frac{\partial v}{\partial t}-D \frac{\partial^{2} v}{\partial x^{2}}<0
$$

with initial condition

$$
v(x, 0)=n(x, 0)-\max _{x}(n(x, 0)),
$$

and zero-flux boundary conditions.

By the maximum principle for parabolic PDEs we have that $v$ must achieve its maximum on one of the boundaries $x=0, x=L$ or $t=0$ (Ockendon et al 2003). Suppose that $v$ takes its maximum value on $x=0$ for some $t=t_{1}>0$. Since $n_{x}\left(0, t_{1}\right)=$ 0 by the zero-flux boundary condition and since $M_{x}\left(t_{1}\right)=0$, as $M$ is independent of $x$, 
this implies that $u_{x}\left(0, t_{1}\right)=0$ and so $v_{x}\left(0, t_{1}\right)=0$. Therefore, in order for this point to be a maximum, we require both that $v_{t}\left(0, t_{1}\right)=0$ and $v_{x x}\left(0, t_{1}\right) \leq 0$. This implies that $v_{t}\left(0, t_{1}\right)-D v_{x x}\left(0, t_{1}\right) \geq 0$, which contradicts (51). Therefore, the maximum cannot lie on the boundary $x=0$. Using a similar argument we can show that the maximum cannot lie on the boundary $x=L$. Therefore, the maximum must lie on the boundary $t=0$. As a check we can see that if the maximum lies on the boundary $t=0$ for some $x=x_{1}$, where $0<x_{1}<L$, then we must have that $v_{t}\left(x_{1}, 0\right) \leq 0, v_{x}\left(x_{1}, 0\right)=0$ and $v_{x x}\left(x_{1}, 0\right) \leq 0$. Thus (51) may be satisfied, provided $\left|v_{t}\left(x_{1}, 0\right)\right|>D\left|v_{x x}\left(x_{1}, 0\right)\right|$.

Since $v(x, t)$ takes its maximum value on $t=0$ this means that $v(x, t) \leq \max _{x}(v(x, 0))=$ $\max _{x}\left(n(x, 0)-\max _{x}(n(x, 0))\right)=\max _{x}(n(x, 0))-\max _{x}(n(x, 0))=0$. Therefore $u \leq 0$, which implies that $n \leq M$. Therefore, by 47), we have that at steady-state $n \leq \kappa F$. Taking the limit as $\beta$ tends to zero, we find $n \leq \kappa \max _{x, t}(f(x, t))=\kappa \max _{x, t}\left(k_{4} n_{h}+\right.$ $\left.\alpha k_{2} n_{o}\right) \leq \kappa \alpha k_{2} n_{T}=\frac{\alpha k_{2} n_{T}}{k_{3}} \approx 4 n_{T} \times 10^{-4}$. This bound was found to be satisfied in all simulations.

\section{Appendix B: Asymptotic Analysis}

In the absence of $\mathrm{Ngb}$, equations (21)-23 reduce to a single equation for oxygen:

$$
0=\frac{\mathrm{d}^{2} c}{\mathrm{~d} x^{2}}-\frac{Q_{i} c}{\gamma+c},
$$

for $Q_{i}\left(1, \ldots, 7^{*}\right)$.

In layers 2 and $4, Q_{2}=Q_{4}=0$, so that an exact analytical solution to equation (53) may be obtained. In all other layers, $Q_{i}$ is strictly positive, so that an exact analytical solution cannot be derived. In these layers we look instead for a leading order solution. Since the value of $Q_{i}$ is not, in general, continuous between layers, we cannot use standard matching techniques to construct a solution which is valid across all layers. Instead we use patching, ensuring that the solution in each layer satisfies the boundary conditions between it and the adjoining layers (see Bender and Orszag 1999. pgs. 335-336 for a discussion of patching).

We construct an asymptotic expansion for $c(x)$ :

$$
c(x) \sim c_{0}(x)+\varepsilon c_{1}(x)+O\left(\varepsilon^{2}\right),
$$

choosing $\varepsilon=0.1$, so as to provide a clear separation between the various scales, and introduce $\gamma^{*}=\varepsilon^{-2} \gamma$ so that $\gamma^{*}=O(1)$. We also note that $Q_{i}=O(1)$ for $i \in$ $\left\{1,3,5,6^{*}, 7^{*}\right\}$ and that $Q_{2}=Q_{4}=0$. Applying the scaling on $\gamma$, equation (53) becomes

$$
0=\frac{\mathrm{d}^{2} c}{\mathrm{~d} x^{2}}-\frac{Q_{i} c}{\varepsilon^{2} \gamma^{*}+c}
$$

We now consider the leading order solution to equation (55) within each layer, grouping layers that have the same scaling. 
Layer 1

In layer $1, c=O(1)$, as can be seen in Figure 9 , and equation (55) supplies, at leading order,

$$
c(x) \sim c_{0}(x)=\frac{Q_{1} x^{2}}{2}+A_{1} x+B_{1},
$$

where $A_{1}$ and $B_{1}$ are constants.

\section{Layers 2 and 4}

In layers 2 and $4, Q_{2}=Q_{4}=0$, so that equation 55 can be solved exactly to yield

$$
c(x)=A_{i} x+B_{i},
$$

for $i=2,4$, where $A_{i}$ and $B_{i}$ are constants.

\section{Layers 3, 5 and $7^{*}$}

In layers 3,5 and $7^{*}, c=O(\varepsilon)$, as can be seen in Figure 9 Rescaling oxygen as $c=\varepsilon c^{*}$, we then rescale $x$ as $x=\varepsilon^{1 / 2} x_{i}^{*}+L_{i-1}$ in order to achieve a dominant balance, so that, after returning to our original scaling on $c$ and $x$ we have, at leading order,

$$
c(x) \sim \frac{Q_{i} x^{2}}{2}+A_{i} x+B_{i},
$$

for $i \in\left\{3,5,7^{*}\right\}$, where $A_{i}$ and $B_{i}$ are constants.

\section{Layer 6*}

The situation in layer $6^{*}$ is less straightforward. On the left- and right-hand sides of layer $6^{*}, c=O(\varepsilon)$, whereas, toward the centre of the layer, in a neighbourhood around the local minimum, $c=O\left(\varepsilon^{2}\right)$. Where $c=O(\varepsilon)$, we could scale $c$ and $x$ as in layers 3, 5 and $7^{*}$; however, in the central region, where $c=O\left(\varepsilon^{2}\right)$, we would regain equation 53 in the dominant balance after dropping the stars. Since seeking leading order solutions does not allow us to avoid dealing with equation (53), we instead retain equation 53 across the whole of layer $6^{*}$ and use quadrature methods to derive approximate analytical solutions for the oxygen profile in this layer.

We will derive separate approximations to the oxygen profile for the left-hand side, centre and right-hand side of layer $6^{*}$, in order to account for the variation in $c$ between $O(\varepsilon)$ and $O\left(\varepsilon^{2}\right)$. We will also derive approximations to the minimum oxygen concentration in layer $6^{*}, c_{\text {min }}$, and its position, $x_{\text {min }}$.

Multiplying equation (53) by $\mathrm{d} c / \mathrm{d} x$ and integrating between $x_{\min }$ and $x$, we find that

$$
\frac{\mathrm{d} c}{\mathrm{~d} x}= \pm \sqrt{2 Q_{6^{*}}} \sqrt{c-c_{\min }-\gamma \log \left(\frac{\gamma+c}{\gamma+c_{\min }}\right)}
$$


where we take the positive (negative) root to the right (left) of $x_{\min }$, since the gradient of the oxygen profile is positive (negative) there. We note that the values of $c_{\min }$ and $x_{\text {min }}$ are unknown at this stage.

We begin by seeking the left-hand and right-hand approximations near $L_{5}$ and $L_{6}$ * respectively. For the left-hand approximation, we integrate equation (59) between $x$ and $L_{5}$ to obtain

$$
\int_{c(x)}^{c_{L}}\left(s-c_{\text {min }}-\gamma \log \left(\frac{\gamma+s}{\gamma+c_{\text {min }}}\right)\right)^{-\frac{1}{2}} \mathrm{~d} s=\sqrt{2 Q_{6^{*}}}\left(x-L_{5}\right),
$$

where $c_{L}=c\left(x=L_{5}\right)$ is unknown at this stage. Since $c \approx c_{L}$ in the left-hand region, where $c_{L}=O(\varepsilon)$, and since $c_{\text {min }}=O\left(\varepsilon^{2}\right)$ and $\gamma=O\left(\varepsilon^{2}\right)$ (see Figure 9 for values of $c_{L}$ and $c_{\text {min }}$ ), we may approximate the integrand by $s^{-\frac{1}{2}}$ to obtain the left-hand approximation as

$$
c(x) \approx \frac{Q_{6^{*}}}{2}\left(L_{5}-x\right)^{2}+\sqrt{2 Q_{6^{*}} c_{L}}\left(L_{5}-x\right)+c_{L} .
$$

In a similar way, we obtain the right-hand approximation:

$$
c(x) \approx \frac{Q_{6^{*}}}{2}\left(x-L_{6^{*}}\right)^{2}+\sqrt{2 Q_{6^{*}} c_{R}}\left(x-L_{6^{*}}\right)+c_{R},
$$

where $c_{R}=c\left(x=L_{6^{*}}\right)$ is unknown at this stage. Both $c_{L}$ and $c_{R}$ may be found by applying the boundary conditions as described at the end of this section.

To derive the central approximation, valid in the neighbourhood of $x_{\text {min }}$, we integrate equation (59) between $x_{\text {min }}$ and $x$. Writing $\log \left((\gamma+s) /\left(\gamma+c_{\text {min }}\right)\right)=\log (1+$ $\left.\left(s-c_{\min }\right) /\left(\gamma+c_{\min }\right)\right)$, we expand the integrand (which is the same as in equation (60) ) about $s=c_{\text {min }}$, in powers of $\left(s-c_{\text {min }}\right) /\left(\gamma+c_{\text {min }}\right)$, where $s$ is the variable of integration and $\left|\left(s-c_{\text {min }}\right) /\left(\gamma+c_{\text {min }}\right)\right| \ll 1$. Retaining only the first term and neglecting higher order terms, we obtain the central solution:

$$
c(x) \approx\left(1+\frac{Q_{6^{*}}\left(x_{\min }-x\right)^{2}}{2\left(\gamma+c_{\text {min }}\right)}\right) c_{\text {min }},
$$

in which $x_{\min }$ and $c_{\min }$ are presently unknown.

To determine $x_{\min }$, we integrate equation 59 between the limits $x_{\min }$ and $L_{5}$, and $x_{\min }$ and $L_{6^{*}}$, to obtain the following pair of equations:

$$
\begin{aligned}
& \int_{c_{\text {min }}}^{c_{L}}\left(s-c_{\text {min }}-\gamma \log \left(\frac{\gamma+s}{\gamma+c_{\text {min }}}\right)\right)^{-\frac{1}{2}} \mathrm{~d} s=\sqrt{2 Q_{6^{*}}}\left(x_{\text {min }}-L_{5}\right), \\
& \int_{c_{\text {min }}}^{c_{R}}\left(s-c_{\text {min }}-\gamma \log \left(\frac{\gamma+s}{\gamma+c_{\text {min }}}\right)\right)^{-\frac{1}{2}} \mathrm{~d} s=\sqrt{2 Q_{6^{*}}}\left(L_{6^{*}}-x_{\text {min }}\right) .
\end{aligned}
$$

Subtracting equation (64) from equation 65), and noting that $\min \left(c_{L}, c_{R}\right)<s<$ $\max \left(c_{L}, c_{R}\right)$ in the integrand, where $c_{L}=O(\varepsilon), c_{R}=O(\varepsilon), c_{\min }=O\left(\varepsilon^{2}\right)$ and $\gamma=$ 
$O\left(\varepsilon^{2}\right)$ (see Figure 9 for values of $c_{L}, c_{R}$ and $c_{\min }$ ), we may approximate the integrand by $s^{-\frac{1}{2}}$ to obtain

$$
x_{\min } \approx \frac{L_{5}+L_{6^{*}}}{2}-\left(\frac{\sqrt{c_{R}}-\sqrt{c_{L}}}{\sqrt{2 Q_{6^{*}}}}\right) .
$$

We obtain an implicit expression for $c_{m i n}$, by substituting for $x_{\min }$ from equation 66 into equation 64]:

$$
\int_{c_{\text {min }}}^{c_{L}}\left(s-c_{\text {min }}-\gamma \log \left(\frac{\gamma+s}{\gamma+c_{\min }}\right)\right)^{-\frac{1}{2}} \mathrm{~d} s \approx \sqrt{\frac{Q_{6^{*}}}{2}}\left(L_{6^{*}}-L_{5}\right)-\left(\sqrt{c_{R}}-\sqrt{c_{L}}\right),
$$

where the integral must be calculated numerically. With $x_{\min }$ and $c_{\min }$ specified by (66) and 677) respectively, we can use equation (63) to calculate the central solution.

\section{Using Iteration to Improve Accuracy}

We can iteratively improve the accuracy of our approximation using the exact derivative, given by equation (59), in the oxygen-flux boundary conditions at $x=L_{6^{*}}$ and $x=L_{7^{*}}$, given by equation $[20$, to yield:

$$
\begin{aligned}
& 0=\sqrt{2 Q_{6^{*}}} \sqrt{c_{L}-c_{\text {min }}-\gamma \log \left(\frac{\gamma+c_{L}}{\gamma+c_{\min }}\right)}+\left(Q_{5} L_{5}+A_{5}\left(c_{L}\right)\right)-\hat{h}_{5}\left(c_{v}-c_{L}\right), \\
& 0=\sqrt{2 Q_{6^{*}}} \sqrt{c_{R}-c_{\min }-\gamma \log \left(\frac{\gamma+c_{R}}{\gamma+c_{\min }}\right)}+Q_{7^{*}}\left(L_{7^{*}}-L_{6^{*}}\right)-\hat{h}_{6^{*}}\left(c_{v}-c_{R}\right) .
\end{aligned}
$$

The constant $c_{\min }$ takes the value calculated by solving (67), using the original values of $c_{L}$ and $c_{R}$, whilst the constant $A_{5}$ is a function of $c_{L}$, given by:

$$
A_{5}\left(c_{L}\right)=\frac{1}{L_{5}}\left[c_{L}-c_{c}-\frac{1}{2}\left(Q_{5}\left(L_{5}^{2}+L_{4}^{2}\right)+Q_{3}\left(L_{2}^{2}-L_{3}^{2}\right)-Q_{1} L_{1}^{2}\right)\right] .
$$

Equations 68 - 69) can be solved numerically, using the Matlab routine fsolve, to find updated values for $c_{L}$ and $c_{R}$. The updated $c_{L}$ and $c_{R}$ can then be used to calculate an updated value for $c_{\min }$, using equation (67). We may then repeat the iteration, using the updated value of $c_{\min }$ in equations 68$)-(70)$, to find new values for $c_{L}$ and $c_{R}$. Once the solution has converged, the final values of $c_{L}$ and $c_{R}$ can then be used to calculate improved values for $A_{1}, \ldots, A_{5}, B_{7^{*}}, x_{\min }$ and $c_{\min }$, by applying the boundary conditions to equation (41) as described in Section 4.3 and using equations (66) and 677.

It was found that iteration results in a small improvement in the accuracy of the approximation and that the solution does not change significantly (by $O\left(10^{-4}\right)$ or greater) after the third iteration. Therefore, we use the parameters generated by the third iteration for the approximate solution in Section 4.3 


\section{References}

Alder VA, Cringle SJ, Constable IJ (1983) The retinal oxygen profile in cats. Invest Ophthalmol Vis Sci 24(1):30-36

Anderson B (1968) Ocular effects of changes in oxygen and carbon dioxide tension. Trans Am Ophthalmol Soc 66:423-474

Anderson B, Saltzman HA (1964) Retinal oxygen utilization measured by hyperbaric blackout. Arch Ophthalmol 72(6):792-795

Bender CM, Orszag SA (1999) Advanced Mathematical Methods for Scientists and Engineers I: Asymptotic Methods and Perturbation Theory. Springer

Bentmann A, Schmidt M, Reuss S, Wolfrum U, Hankeln T, Burmester T (2005) Divergent distribution in vascular and avascular mammalian retinae links neuroglobin to cellular respiration. J Biol Chem 280(21):20,660-20,665

Birol G, Wang S, Budzynski E, Wangsa-Wirawan ND, Linsenmeier RA (2007) Oxygen distribution and consumption in the macaque retina. Am J Physiol Heart Circ Physiol 293(3):H1696-H1704

Braun RD, Linsenmeier RA, Goldstick TK (1995) Oxygen consumption in the inner and outer retina of the cat. Invest Ophthalmol Vis Sci 36(3):542-554

Burmester T, Hankeln T (2004) Neuroglobin: A respiratory protein of the nervous system. News Physiol Sci 19(3):110-113

Burmester T, Hankeln T (2009) What is the function of neuroglobin? J Exp Biol 212(10):1423-1428

Burmester T, Weich B, Reinhardt S, Hankeln T (2000) A vertebrate globin expressed in the brain. Nature 407(6803):520-523

Burmester T, Ebner B, Weich B, Hankeln T (2002) Cytoglobin: A novel globin type ubiquitously expressed in vertebrate tissues. Mol Biol Evol 19(4):416-421

Chan G, Balaratnasingam C, Yu PK, Morgan WH, McAllister IL, Cringle SJ, Yu DY (2012) Quantitative morphometry of perifoveal capillary networks in the human retina. Invest Ophthalmol Vis Sci 53(9):5502-5514

Costa LE, Mendez G, Boveris A (1997) Oxygen dependence of mitochondrial function measured by high-resolution respirometry in long-term hypoxic rats. Am J Physiol 273(3):C852-C858

Cringle SJ, Yu DY (2002) A multi-layer model of retinal oxygen supply and consumption helps explain the muted rise in inner retinal $\mathrm{PO}(2)$ during systemic hyperoxia. Comp Biochem Physiol 132(1):61-66

Dollery CT, Bulpitt CJ, Kohner EM (1969) Oxygen supply to the retina from the retinal and choroidal circulations at normal and increased arterial oxygen tensions. Invest Ophthalmol Vis Sci 8(6):588-594

Fago A, Hundahl C, Malte H, Weber RE (2004b) Functional properties of neuroglobin and cytoglobin. insights into the ancestral physiological roles of globins. IUBMB Life 56(11-12):689-696

Gillies MC, Su T, Naidoo D (1995) Electrical resistance and macromolecular permeability of retinal capillary endothelial cells in vitro. Curr Eye Res 14(6):435-442

Goldman D (2008) Theoretical models of microvascular oxygen transport to tissue. Microcirculation 15(8):795-811 
Hamdane D, Kiger L, Dewilde S, Green BN, Pesce A, Uzan J, Burmester T, Hankeln T, Bolognesi M, Moens L, Marden MC (2003) The redox state of the cell regulates the ligand binding affinity of human neuroglobin and cytoglobin. J Biol Chem 278(51):51,713-51,721

Hardarson SH, Stefánsson E (2010) Oxygen saturation in central retinal vein occlusion. Am J Ophthalmol 150(6):871-875

Hardarson SH, Basit S, Jonsdottir TE, Eysteinsson T, Halldorsson GH, Karlsson RA, Beach JM, Benediktsson JA, Stefansson E (2009) Oxygen saturation in human retinal vessels is higher in dark than in light. Invest Ophthalmol Vis Sci 50(5):23082311

Haugh L, Linsenmeier R, Goldstick T (1990) Mathematical models of the spatial distribution of retinal oxygen tension and consumption, including changes upon illumination. Ann Biomed Eng 18:19-36

Jürgens KD, Peters T, Gros G (1994) Diffusivity of myoglobin in intact skeletal muscle cells. Proc Natl Acad Sci 91(9):3829-3833

Keener J, Sneyd J (1998) Mathematical Physiology. Springer

Kiger L, Uzan J, Dewilde S, Burmester T, Hankeln T, Moens L, Hamdane D, BaudinCreuza V, Marden MC (2004) Neuroglobin ligand binding kinetics. IUBMB Life 56(11-12):709-719

Kohen R, Nyska A (2002) Invited review: Oxidation of biological systems: Oxidative stress phenomena, antioxidants, redox reactions, and methods for their quantification. Toxicol Pathol 30(6):620-650

Kur J, Newman EA, Chan-Ling T (2012) Cellular and physiological mechanisms underlying blood flow regulation in the retina and choroid in health and disease. Prog Retin Eye Res 31(5):377-406

Linsenmeier RA (1986) Effects of light and darkness on oxygen distribution and consumption in the cat retina. J Gen Physiol 88(4):521-542

Linsenmeier RA, Braun RD (1992) Oxygen distribution and consumption in the cat retina during normoxia and hypoxemia. J Gen Physiol 99(2):177-197

Linsenmeier RA, Padnick-Silver L (2000) Metabolic dependence of photoreceptors on the choroid in the normal and detached retina. Invest Ophthalmol Vis Sci 41(10):3117-3123

Linsenmeier RA, Yancey CM (1989) Effects of hyperoxia on the oxygen distribution in the intact cat retina. Invest Ophthalmol Vis Sci 30(4):612-618

McGuire BJ, Secomb TW (2001) A theoretical model for oxygen transport in skeletal muscle under conditions of high oxygen demand. J Appl Physiol 91(5):2255-2265

Ockendon J, Howison S, Lacey A, Movchan A (2003) Applied Partial Differential Equations, revised edn. Oxford University Press

Ostojić J, Sakaguchi DS, de Lathouder Y, Hargrove MS, Trent JT, Kwon YH, Kardon RH, Kuehn MH, Betts DM, Grozdanić S (2006) Neuroglobin and cytoglobin: Oxygen-binding proteins in retinal neurons. Invest Ophthalmol Vis Sci 47(3):1016-1023

Ostojić J, Grozdanić SD, Syed NA, Hargrove MS, Trent JT, Kuehn MH, Kwon YH, Kardon RH, Sakaguchi DS (2008) Patterns of distribution of oxygenbinding globins, neuroglobin and cytoglobin in human retina. Arch Ophthalmol 126(11):1530-1536 
Oyster CW (1999) The Human Eye: Structure and Function. Sinauer Associates Inc. Padnick-Silver L, Linsenmeier RA (2003) Effect of acute hyperglycemia on oxygen and oxidative metabolism in the intact cat retina. Invest Ophthalmol Vis Sci 44(2):745-750

Pournaras CJ, Rungger-Brändle E, Riva CE, Hardarson SH, Stefansson E (2008) Regulation of retinal blood flow in health and disease. Prog Retin Eye Res 27(3):284 330

Rajendram R, Rao NA (2007) Neuroglobin in normal retina and retina from eyes with advanced glaucoma. Br J Ophthalmol 91(5):663-666

Richmond KN, Shonat RD, Lynch RM, Johnson PC (1999) Critical PO(2) of skeletal muscle in vivo. Am J Physiol Heart Circ Physiol 277(5):H1831-H1840

Roh HD, Goldstick TK, Linsenmeier RA (1990) Spatial variation of the local tissue oxygen diffusion coefficient measured in situ in the cat retina and cornea. Adv Exp Med Biol 277:127-136

Schmidt M, Giessl A, Laufs T, Hankeln T, Wolfrum U, Burmester T (2003) How does the eye breathe? J Biol Chem 278(3):1932-1935

Schmidt M, Laufs T, Reuss S, Hankeln T, Burmester T (2005) Divergent distribution of cytoglobin and neuroglobin in the murine eye. Neurosci Lett 374(3):207-211

Stefánsson E (1988) Retinal oxygen tension is higher in light than dark. Pediatr Res 23:5-8

Swaroop A, Kim D, Forrest D (2010) Transcriptional regulation of photoreceptor development and homeostasis in the mammalian retina. Nat Rev Neurosci 11:563576

Tan PEZ, Yu PK, Balaratnasingam C, Cringle SJ, Morgan WH, McAllister IL, Yu DY (2012) Quantitative confocal imaging of the retinal microvasculature in the human retina. Invest Ophthalmol Vis Sci 53(9):5728-5736

Törnquist P, Alm A, Bill A (1990) Permeability of ocular vessels and transport across the blood-retinal-barrier. Eye 4(2):303-309

Trent JT, Hargrove MS (2002) A ubiquitously expressed human hexacoordinate hemoglobin. J Biol Chem 277(22):19,538-19,545

Wangsa-Wirawan ND, Linsenmeier RA (2003) Retinal oxygen: Fundamental and clinical aspects. Arch Ophthalmol 121(4):547-557

Wilson DF, Rumsey WL, Green TJ, Vanderkooi JM (1988) The oxygen dependence of mitochondrial oxidative phosphorylation measured by a new optical method for measuring oxygen concentration. J Biol Chem 263(6):2712-2718

Yu DY, Cringle SJ (2001) Oxygen distribution and consumption within the retina in vascularised and avascular retinas and in animal models of retinal disease. Prog Retin Eye Res 20(2):175-208

Yu DY, Cringle SJ (2002) Outer retinal anoxia during dark adaptation is not a general property of mammalian retinas. Comp Biochem Physiol 132(1):47-52

Yu DY, Cringle SJ (2005) Retinal degeneration and local oxygen metabolism. Exp Eye Res 80(6):745-751

Yu DY, Cringle SJ, Alder VA, Su EN (1994) Intraretinal oxygen distribution in rats as a function of systemic blood pressure. Am J Physiol Heart Circ Physiol 267(6):H2498-H2507 
Yu DY, Cringle SJ, Alder V, Su EN (1999) Intraretinal oxygen distribution in the rat with graded systemic hyperoxia and hypercapnia. Invest Ophthalmol Vis Sci 40(9):2082-2087

Yu DY, Cringle SJ, Su EN, Yu PK (2000) Intraretinal oxygen levels before and after photoreceptor loss in the RCS rat. Invest Ophthalmol Vis Sci 41(12):3999-4006

Yu DY, Cringle S, Valter K, Walsh N, Lee D, Stone J (2004) Photoreceptor death, trophic factor expression, retinal oxygen status, and photoreceptor function in the P23H rat. Invest Ophthalmol Vis Sci 45(6):2013-2019

Yu DY, Cringle SJ, Su EN (2005) Intraretinal oxygen distribution in the monkey retina and the response to systemic hyperoxia. Invest Ophthalmol Vis Sci 46(12):47284733 